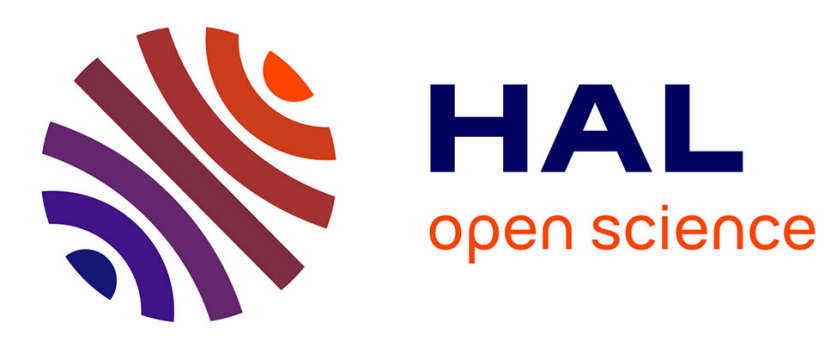

\title{
Optic flow selectivity in the macaque parieto-occipital sulcus
}

Sabrina Pitzalis, Fadila Hadj-Bouziane, Giulia Dal Bò, Carole Guedj, Francesca Strappini, Martine Meunier, Alessandro Farnè, Patrizia Fattori, Claudio Galletti

\section{To cite this version:}

Sabrina Pitzalis, Fadila Hadj-Bouziane, Giulia Dal Bò, Carole Guedj, Francesca Strappini, et al.. Optic flow selectivity in the macaque parieto-occipital sulcus. Brain Structure and Function, 2021, $10.1007 / \mathrm{s} 00429-021-02293-\mathrm{w}$. hal-03375675

\section{HAL Id: hal-03375675 \\ https://hal.science/hal-03375675}

Submitted on 14 Oct 2021

HAL is a multi-disciplinary open access archive for the deposit and dissemination of scientific research documents, whether they are published or not. The documents may come from teaching and research institutions in France or abroad, or from public or private research centers.
L'archive ouverte pluridisciplinaire HAL, est destinée au dépôt et à la diffusion de documents scientifiques de niveau recherche, publiés ou non, émanant des établissements d'enseignement et de recherche français ou étrangers, des laboratoires publics ou privés. 


\section{Optic Flow selectivity in the macaque parieto-occipital sulcus}

Sabrina Pitzalis*1,2,\$,a, Fadila Hadj-Bouziane ${ }^{3,4, \S}$, Giulia Dal Bo ${ }^{5}$, Carole Guedj ${ }^{3,4}$, Francesca Strappini ${ }^{6}$, Martine Meunier $^{3,4}$, Alessandro Farnè ${ }^{3,4}$, Patrizia Fattori ${ }^{5, b}$ and Claudio Galletti ${ }^{5, c}$

${ }^{1}$ Department of Movement, Human and Health Sciences, University of Rome "Foro Italico", Italy

2 Department of Cognitive and Motor Rehabilitation and Neuroimaging, Santa Lucia Foundation (IRCCS Fondazione Santa Lucia), Rome, Italy

${ }^{3}$ Integrative Multisensory Perception Action \& Cognition Team (ImpAct), INSERM U1028, CNRS UMR5292, Lyon Neuroscience Research Center (CRNL), 69000 Lyon, France

${ }^{4}$ University of Lyon 1, 69000 Lyon, France

${ }^{5}$ Department of Biomedical and Neuromotor Sciences, University of Bologna, Bologna, Italy

${ }^{6}$ Department of Neurobiology, Weizmann Institute of Science, Rehovot, Israel

$\S$ : Both authors contributed equally to this work.

\section{ORCID ID:}

a https://orcid.org/0000-0002-4445-0391

b https://orcid.org/0000-0002-0079-3755

c https://orcid.org/0000-0001-8927-3408

\section{*Correspondence:}

Sabrina Pitzalis, PhD

Department of Movement, Human and Health Sciences

University of Rome "Foro Italico"

00194 Rome (Italy)

phone: 0039-06.36733.383

fax: 0039- 06.36733.387

e-mail: sabrina.pitzalis@uniroma4.it 


\begin{abstract}
In humans, several neuroimaging studies have demonstrated that passive viewing of optic flow stimuli activates higher-level motion areas, like V6 and the cingulate sulcus visual area (CSv). In macaque, there are few studies on the sensitivity of V6 and CSv to egomotion compatible optic flow. The only fMRI study on this issue revealed selectivity to egomotion compatible optic flow in macaque CSv but not in V6 (Cotterau et al, 2017, but see Fan et al. 2015). Yet, it is unknown whether monkey visual motion areas MT+ and V6 display any distinctive fMRI functional profile relative to the optic flow stimulation, as it is the case for the homologous human areas (Pitzalis et al., 2010). Here, we described the sensitivity of the monkey brain to two motion stimuli (Radial Rings and Flow Fields) originally used in humans to functionally map the motion middle temporal area MT+ (Tootell et al. 1995a,b) and the motion medial parietal area V6 (Pitzalis et al. 2010), respectively. In both animals, we found regions responding only to optic flow or Radial Rings stimulation, and regions responding to both stimuli. A region in the parieto occipital sulcus (likely including V6) was one of the most highly selective area for coherently moving fields of dots, further demonstrating the power of this type of stimulation to activate $V 6$ in both humans and monkeys. We did not find any evidence that putative macaque CSv responds to Flow Fields.
\end{abstract}

Keywords: Monkey fMRI, Flow Fields, parieto occipital cortex, Egomotion 


\section{Introduction}

Neural representation of optic flow has been extensively studied in macaques and humans.

In monkeys, several single unit and neuroimaging studies have demonstrated that passive viewing of optic flow stimuli activates some higher-level motion areas, including the middle superior temporal (MST) area (Saito et al. 1986; Tanaka et al. 1989; Orban et al. 1992; Duffy, 1998; Vanduffel et al., 2001; De Angelis et al., 2012) and the superior temporal polysensory (STPm) area (Nelissen et al., 2006) in the superior temporal sulcus, the ventral intraparietal (VIP) area in the intraparietal sulcus (Duhamel et al., 1998), area PEc in the anterior precuneus (Raffi et al. 2002, 2011) and the visual posterior Sylvian (VPS) area in the parieto-insular vestibular cortex (Chen et al., 2011a,b).

In humans, responses to optic flow have been demonstrated in a larger cortical network of temporal, parietal, insular and cingulate regions. This network includes MST (MT+; Tootell et al., 1995a,b; Morrone et al., 2000; Pitzalis et al., 2013c), VIP (Bremmer et al., 2001; Sereno and Huang, 2006; Cardin and Smith, 2010), the newly defined human homologue of area PEc (Pitzalis et al., 2019), and the parieto-insular vestibular cortex (PIVC or PIC: Claeys et al., 2003; Cardin and Smith, 2010), like in monkeys, but also V6 in the parieto occipital sulcus (Pitzalis et al., 2010), two regions in the cingulate sulcus (cingulate visual area, Csv, and posterior cingulate area, Pc or pCi; Wall and Smith 2008; Cardin and Smith, 2010; Serra et al., 2019), and p2v in the dorsal post-central gyrus (Cardin and Smith, 2010). Some of these human areas, like V6 and CSv, are now consolidated egomotion regions, whose functional profile has been studied in many recent neuroimaging studies from our and other laboratories (Fisher et al., 2012a; Furlan et al., 2014; Pitzalis et al., 2013b, 2020; Greenlee et al., 2016; Serra et al., 2019).

Surprisingly, we still know very little about the functional role played by these two medial areas, V6 and CSv, in the monkey. While most of the $\mathrm{fMRI}$ studies focused their investigations mainly on the temporal and dorsal intraparietal cortices, motion-related activations on the medial wall received scarce attention. CSv was first defined in the human brain (Wall e Smith, 2008) and only very recently identified in macaque (Cotterau et al., 2017), although with a less striking functional profile with respect to that seen in human, where CSv is one of the most systematically activated areas for egomotion stimuli (e.g., Serra et al., 2019). On the contrary, area V6 was originally described in macaque (Galletti et al., 1996, 1999) and then also reported in human (Pitzalis et al. 2006, 2010; Cardin and Smith, 2010). Using simple visual stimuli like light/dark borders moving across the visual field, Galletti and coworkers (Galletti et al., 1996, 1999) found that the majority of V6 neurons were motion and direction sensitive and responded well to large stimuli. The presence of strong directional sensitivity, together with the wide representation of the far periphery, suggested that V6 "could be engaged in the analysis of flow field resulting from self-motion" (see Galletti et al., 1999). However, in spite of this likely prediction, and of the already proved sensitivity to the optical flow of human V6 (Pitzalis et al. 2010, 2020), the sensitivity of macaque $V 6$ cells to optical flow stimulations has not been systematically tested so far. In a single unit study, Fan et al. (2015) found that the majority of V6 neurons were selective for heading defined by optic flow, but the only fMRI study on the neural basis of egomotion in macaque failed to find a V6 preference for egomotion compatible optic flow (Cotterau et al, 2017). The experimental protocol of Cotterau et al. (2017) was similar to that used in their previous human studies in which the responses to a single large optic flow patch were contrasted with responses to an array of nine small similar flow patches. Since the optic flow used by (Cotterau et al, 2017) was different from that used in human by our group (Pitzalis et al., 2010, 2020), here we sought to test whether macaque V6 is sensitive to the same optic flow (Flow Fields) we used in human to localize area V6. In addition, we want to verify whether Flow fields are able to activate also CSv in the monkey and all the other egomotion-related areas found in the human brain.

Yet, in humans, we originally found a different functional profile between the two motion areas V6 and MT+ (Pitzalis et al., 2010) when optic flows or radial motion stimuli were used (Pitzalis et al., 2012). In particular, we found that V 6 was well activated by coherent motion of dot fields (Flow Fields; see Figure 1B) but not by 
radial motion of low contrast rings (Radial Rings; see Figure $1 \mathrm{~A}$ ); conversely, the MT+ was well activated by Radial Rings (the stimulus classically used to map MT+; Tootell et al., 1995a,b) and showed also a significant response to Flow Fields, although much weaker with respect to Radial Rings (Serra et al., 2019; Sulpizio et al., 2020). Here, we sought to test whether monkey regions in the STS and POs display any distinctive fMRI functional profile relative to optic flow and radial motion stimuli, as it is the case in humans (Pitzalis et al., 2010).

In the present study, we performed an fMRI study in alert fixating macaques using the Flow Fields and Radial Rings stimuli used in the human fMRI studies as functional localizer of areas V6 (Pitzalis et al., 2010) and MT+ (Tootell et al., 1995a,b), respectively. We first conducted a whole-brain analysis to have a general picture of the brain regions involved in both Radial Rings and Flow Fields visual motion stimuli. Then we performed a regional analysis to study the functional response profile of each motion region with respect to the two visual motion stimuli used here. We showed that a region in the POs (likely including V6) was one of the most highly selective area for coherently moving fields of dots, further demonstrating the power of this type of stimulation to activate $\mathrm{V} 6$ in both humans and monkeys. We observed a different functional profile between temporal regions along the STS and V6+ which mirrors the functional results observed in the human counterparts.

\section{Materials and Methods}

\section{Subjects}

Two female rhesus monkeys (Macaca mulatta, 6 and 13 years of age, 5 and $7 \mathrm{~kg}$ ) participated in this study. fMRI experiments were carried out in Lyon, in accordance with European Union Directive 2010/63/EU and were approved by French Animal Experimentation Ethics Committee \#42 (CELYNE).

\section{Surgical preparation and behavioral training}

Each monkey was surgically implanted with a plastic head post in aseptic conditions under anesthesia (glycopyrrolate $0.01 \mathrm{mg} / \mathrm{kg}$, i.m. + acepromazine maleate $0.05 \mathrm{ml} / \mathrm{kg}$, i.m. + ketamine $10 \mathrm{mg} / \mathrm{kg}$, i.m.) followed by the gaseous anesthesia (2-chloro-2-(difluoromethoxy)-1,1,1-trifluoroethane 0.5 to $2 \%$ depending on the condition of the animal). The body temperature was controlled throughout the procedure. The animal was intubated and ventilated with a mixture of $30 \% \mathrm{O}_{2}$ and $70 \% \mathrm{~N}_{2} \mathrm{O}$. Tidal volume and respiratory rate were tailored according to each animal (approximately $10 \mathrm{ml} / \mathrm{kg}$ at $20-30$ breaths per minute). An intravenous line was placed in the saphenous vein through which a saline solution was delivered at $5 \mathrm{ml} / \mathrm{kg} / \mathrm{hour}$. The animal was then placed in a stereotaxic frame before the surgery begins. An MRI-compatible plastic head post was implanted on the skull and secured by plastic screws and bone cement. After surgery, the animal got under close supervision for a minimum of 20 days before being involved in experimental procedures. Postoperative pain was controlled by buprenorphine hydrochloride $(0.01 \mathrm{mg} / \mathrm{kg}$, i.m.). Postoperative treatment further consisted of antibiotics (enrofloxacin $5 \mathrm{mg} / \mathrm{kg}$, i.m. for 10 days), and anti-inflammatory agent (tolfenamic acid $4 \mathrm{mg} / \mathrm{kg}$, i.m. for 3 days).

After recovery, alert monkeys were trained to sit in a sphinx position in a plastic restraint chair with their heads fixed, facing a screen on which visual stimuli were presented in complete darkness. Monkeys underwent 2-5 training sessions per week in a mock scanner in order to optimize the experimental setup and familiarize the animals to the primate chair and the fMRI sound. Monkeys were trained to fixate a small central fixation cross to receive a liquid reward. In the reward schedule, to promote longer periods of fixation the frequency of reward delivery increased as the duration of fixation increased. The gaze location was monitored using an infrared pupil tracking system (ISCAN) centered on the right eye. The implant was cleaned after each training session with betadine and hydrogen peroxide.

\section{Visual Stimuli}


Visual stimuli were presented by using a projector (Canon XEED SX60) and displayed on a back-projection screen positioned just outside the magnet bore at $90 \mathrm{~cm}$ distance from the monkeys' face $\left(36^{\circ}\right.$ wide of visual field tested). The animals were head-fixed, seated in a sphynx position within their primate chair. Throughout all scanning runs, stimuli were overlaid with a $0.2^{\circ}$ centrally located fixation cross, on which the monkeys were required to fixate. Presentation ${ }^{\circledR}$ program (Neurobehavioral systems) was used to run the fixation task and control the reward; visual stimuli were presented using Mac OS X.

We used two visual stimuli identical to those used in our previous human study (Pitzalis et al., 2010) in two separate fMRI sessions. In both fMRI experiments, we used 2-condition stimuli (4 epochs of 32-s ON and 32s OFF) described below.

"Low Contrast Expanding and Contracting rings" hereafter called "Radial Rings" (RR) (Figure 1A). This stimulus was originally used by Tootell et al. $(1995 a, b)$ to functionally define the human motion area MT. Stimuli produced by an X11/OpenGL program (original GL code by A. Dale, ported and extended by M. Sereno) were concentric thin light gray rings $(0.2 \mathrm{cycles} / \mathrm{deg}$, duty cycle $=0.2)$ on a slightly darker-gray background, either moving ( $7 \mathrm{deg} / \mathrm{s}-\mathrm{ON}$ period) or stationary (OFF period). During the ON block, the concentric rings periodically contracted and expanded $(1 \mathrm{~s}, 1 \mathrm{~s})$ to avoid generating motion aftereffects during the OFF block. The stimulus luminance contrast was low ( 1.5\%) as described in Tootell et al. $(1995 \mathrm{a}, \mathrm{b})$ to better isolate human MT+ with respect to the neighboring visual areas. The average luminance of the stimulus was $61 \mathrm{~cd} / \mathrm{m} 2$. In the previous experiments on human subjects (Pitzalis et al., 2010), participants reported after the session that the stimulus produced no illusory perception of self-motion (vection).

\begin{abstract}
"High-contrast Flow Fields" hereafter called "Flow Fields" (FF) (see Figure 1B). The same X11/OpenGL software (Dale and Sereno) produced 16-s blocks of coherent dot field motion contrasted with scrambled motion. A new field of white dots was generated every $500 \mathrm{~ms}$ (dot size $0.4 \times 0.4 \mathrm{deg}^{2}$ ). Dots immediately began to move along a trajectory so as to generate a coherent movement on a plane. The pattern motion was chosen randomly for that 500-ms period from a continuum ranging from dilation to outward spiral, to rotation, to inward spiral, to contraction. The center of the movement was jittered from flow to flow, and the speed varied within a small range. During the scrambled OFF period, dots and their movement vectors were generated as during the coherent $\mathrm{ON}$ periods except that each dot trajectory was rotated by a random angle around the pattern center before execution. This scrambled the coherency of movement (at a given point, dots moved in different directions) but preserved the speed gradient (central dots still moved slower than peripheral dots). In the previous paper on human subjects (Pitzalis et al., 2010), we reported that both the wide field $\left(100^{\circ} \times 80^{\circ}\right)$ and the standard versions $\left(23^{\circ} \times 12^{\circ}\right)$ of this optic flow stimulus induced a compelling vection sensation according to subject verbal reports after the session.
\end{abstract}

\title{
Scanning
}

Before each scan session, $10-12 \mathrm{mg} / \mathrm{kg}$ of an exogenous contrast agent [monocrystalline iron oxide nanocolloid (MION)] was injected into the saphenous vein to increase the contrast/noise ratio and to optimize the localization of fMRI signals (e.g., Vanduffel et al., 2001).

Imaging data were collected on a 1.5T MAGNETOM (Sonata, Siemens AG, Erlangen, Germany) horizontal scanner ( $60 \mathrm{~cm}$ diameter of the bore). Functional data from the whole brain were acquired with a circular 9$\mathrm{cm}$ custom-made, receive-only surface RF coil (1 channel, RAPID Biomed) positioned immediately over the head using gradient-recalled echo-planar imaging.

For both Radial Rings and Flow Fields we acquired 20 contiguous coronal slices [voxel resolution $2 \times 2 \times 2 \mathrm{~mm}$; repetition time (TR) $2 \mathrm{~s}$; echo time (TE) $27 \mathrm{~ms}$, flip angle $=90^{\circ}$, field of view $=129 \mathrm{~mm}$, matrix $=64 \times 64,132$ ] Each run of scanning lasted $257 \mathrm{~s}$ with 132 single-shot EPI images per slice (TRs). In each run, the first four volumes were discarded from data analysis in order to achieve a steady-state, and the stimuli started at the beginning of the fifth volume. The imaging data were collected in each monkey over two scanning sessions. In monkey 1 we collected 8 runs in the first session and 25 runs in the second (i.e., 16 fMRI runs for Flow Fields and 17 runs for Radial Rings). In monkey 2 we collected 21 runs in the first session and 13 runs in the second (i.e., $16 \mathrm{fMRI}$ runs for Flow Fields and 18 runs for Radial Rings). 
In separate scan sessions, high-resolution anatomical scans were obtained from each monkey under anesthesia. Each monkey underwent three structural scans (T1-weighted sagittal Magnetization Prepared Rapid Gradient Echo (MPRAGE) sequence: $0.6 \mathrm{~mm}^{3}$ voxels, matrix 256x256, 176 contiguous coronal slices). These anatomical scans were used as an underlay for the functional data and for cortical surface reconstruction. For a detailed description of the scanning procedure see Hadj-Bouziane et al. (2008; 2012; 2014).

\section{Data Analysis}

Anatomical and functional individual MRI data were analyzed using UCSD/UCL FreeSurfer (Dale et al., 1999; Fischl et al., 1999) as described below.

Structural MRI data. The anatomical MRI data acquired from each monkey were used for cortical surface reconstruction using UCSD/UCL FreeSurfer (Dale et al., 1999; Fischl et al., 1999) based on standard procedures described in detail elsewhere (Sereno et al., 1995; Tootell et al., 1997; Hagler and Sereno, 2006; Pitzalis et al., 2006, 2010, 2013d). Briefly, three high-resolution structural images (MPRAGES), obtained from each monkey, were manually registered and averaged. The skull was stripped off by expanding a stiff deformable template out to the dura, the gray/white matter boundary was estimated with a region-growing method, and the result was tessellated to generate a surface that was refined against the MRI data with a deformable template algorithm. By choosing a surface near the gray/white matter border (rather than near the pial surface, where the macrovascular artifact is maximal), we were able to assign activations more accurately to the correct bank of a sulcus. The surface was then unfolded by reducing curvature while minimizing distortion in all other local metric properties. After reconstruction, each hemisphere was then completely flattened using five relaxation cuts: one cut along the calcarine fissure, three equally spaced radial cuts on the medial surface, and one sagittal cut around the temporal lobe.

\section{Functional MRI data}

Voxel-wise analysis. $\mathrm{fMRI}$ data from the two-condition $\mathrm{fMRI}$ experiments were analyzed based on standard procedures described in many previous studies (e.g. Sereno et al., 1995; Pitzalis et al., 2010, 2012, 2013a,c; Strappini et al., 2015). Briefly, the first (pre-magnetization steady state) 4 volumes were discarded. Motion correction and cross-scan alignment were performed using the 3 dvolreg tool of the Analysis of Functional Neurolmages (AFNI) software package (Cox, 1996; http://afni.nimh.nih.gov). Block sequence data were analyzed by voxelwise Fourier transforming the fMRI time series (after removing constant and linear terms). $P$ values were estimated on a voxel-by-voxel basis by constructing an F ratio between 'signal' (response amplitude at stimulus frequency) and 'noise' (amplitude at other frequencies excluding second and third harmonics) with degrees of freedom equal to the number of time points. This analysis is used both in mapping studies (i.e., phase-encoded retinotopic data) and to distinguish between positive and negative-going MR fluctuations in the case of 2-condition stimulus comparisons. In a standard block-design analysis, the phase of the signal at the stimulus frequency is used to represent the amplitude of the response (after masking the data with a significance threshold). We modulated the saturation of the color as a function of the signal amplitude using a sigmoid function. The sigmoid function was arranged so that visibly saturated phase colors begin to emerge from the gray background at a threshold of $p<10^{-2}$. The data at most activated cortical surface points have much higher significance values $\left(p<10^{-5}\right.$ to $\left.10^{-10}\right)$. We used an individually adjusted threshold for each monkey to avoid missing information (false negative). The $F$ ratio was subsequently corrected at $\mathrm{p}<10^{-5}$ using a surface-based cluster correction for multiple comparisons as implemented by surfclust and randsurfclust within the FreeSurfer framework (Hagler et al., 2006). Additional affine transformations that included a small amount of shear were then applied to the functional scans for each subject using blink comparison with the structural images to achieve an exact overlay of the functional data. After Fourier analysis, we applied to all fMRI data a surface-based smoothing (step 3.20) by averaging the values of adjacent vertices, with an iterative algorithm implemented in UCSD/UCL FreeSurfer (Hagler et al., 2006). It has been shown that this method is computationally simpler and more efficient than the iterative smoothing based on the diffusion model (Hagler et al., 2006; Strappini et al., 2017). Note that throughout 
this paper all $\mathrm{fMRI}$ signals have been inverted so that an increase in cerebral blood volume is represented by an increase in signal intensity. Results from this first whole-brain analysis are shown in Figures 2, 3 and 4.

Surface-based Region of Interest (ROI) definition and regional analysis. In the regional approach, we focused our analysis on regions that were significantly activated in at least 3 out of the 4 hemispheres of our two monkeys. This approach drastically reduces the risk of false positives. Since we did not include a retinotopic mapping or an independent a priori ROI definition, we referred to each ROI using anatomical labels instead of the functional subdivisions known to occupy those cortical regions. The anatomical label exclusively refers to the position of the ROI with respect to the sulcal pattern. It is used to differentiate the regions responding to the visual stimulation conditions and should not be intended as a way to introduce a new terminology. Specifically, we defined six regions from Flow Fields condition (4/4 Hs: POs, dSTS, aSTS, pIns, pIPs; 3/4 Hs: dIPs) and three regions from Radial Rings condition (4/4 Hs: mSTS; 3/4 Hs: IOs, Lunate). Table 1 lists all the acronyms used in the paper to indicate brain ROIs. We defined these ROIs on the individual reconstructed monkey cortical surface following a two-steps procedure. For each monkey and experiment, we split the runs into 2 parts. First, we used half of the runs to define a set of individual ROIs (shown in Figure 5) extracting activation peaks from the statistical map and including in each ROI all activated voxels (cluster-corrected $p<$ $10^{-2}$ ) within a maximum distance from the peak. We chose a relaxed threshold to avoid the risk of false negatives, as done also previously by other groups (e.g., Cotterau et al., 2017). Second, in the other half of the runs, we estimated the averaged amplitude of the hemodynamic response for each $\mathrm{FMRI}$ experiment (Flow Fields and Radial Rings) and for each hemisphere in the ROls found with the first-step analysis. Such regional hemodynamic response estimates are shown in the plots of Figures $6 \mathrm{~A}$ and $6 \mathrm{~B}$. This two-steps method minimized the risk of "double-dipping" that arises when the same data is used both for identifying the ROls and for measuring the activity within them. Given the low number of animals, there is no statistical test of generalizability. We therefore present individual data only and focus on regions that were consistently found in the two macaques (in at least three out of four hemispheres). Regional hemodynamic responses were analyzed through a series of one sample $t$-tests to assess for the presence of Radial Rings-related responses in areas defined by Flow Fields (POs, dSTs, aSTS, pIns, pIPs, dIPs) and the presence of Flow Fieldsrelated responses in areas defined by Radial Rings (mSTS, IOs, Lunate). For this analysis, we applied a Bonferroni correction for multiple comparisons $(p=0.05 / N=9$, number of regions; $p=.006)$. P-values are interpreted as significant and Bonferroni-corrected when surviving the calculated threshold. For the regional analysis, runs were selected based on the quality of fixation (average percentage of correct fixation $=86 \%$ ). More importantly, in these runs we did not reveal differences in the quality of fixation evoked by Flow Fields and radial rings stimuli, in any of the 2 monkeys as indicated by the not significant paired t-tests (vs Radial Rings; Monkey 1: $t_{7}=-0.38 ; p=0.71 ;$ Monkey 2: $t_{7}=-0.84 ; p=0.43$ ).

\begin{tabular}{ll}
\hline Macaque ROIs & \\
\hline POs & Parieto Occipital sulcus \\
dSTs & dorsal Superior Temporal sulcus \\
mSTs & middle Superior Temporal sulcus \\
aSTs & anterior Superior Temporal sulcus \\
dIPs & dorsal IntraParietal sulcus \\
pIPs & posterior IntraParietal sulcus \\
pIns & Posterior Insula \\
IOs & Inferior Occipital sulcus \\
Lunate & Lunate sulcus \\
\hline
\end{tabular}

Tab 1. Lists all the acronyms used in the paper to indicate macaque brain surface Regions of Interest (ROIs).

Sensitivity Ratio. In each ROI, specificity of the MION responses to FF versus RR stimuli was quantified by computing a sensitivity ratio with the following formula:

$$
\text { Relative Effect size }=(F F-R R) /(F F+R R)
$$


as the ratio between the difference in the activation between FF and RR and the sum of the activation in FF and RR, averaging together the results across the four hemispheres from both monkeys. Scores equal to zero imply same activation for Flow Fields and Radial Rings, scores greater than zero imply higher activation for Flow Fields than Radial Rings, while scores smaller than zero imply higher activation for Radial Rings than Flow Fields (for a similar approach see Cardin and Smith, 2010; Pitzalis et al., 2013c; Cottereau et al., 2017). Results on the sensitivity ratio computed in each $\mathrm{ROI}$ is shown in Figure 8.

Results Visualization. These individual results (both voxel-wise and ROI maps) were projected not onto an anatomical template (as typically done in fMRI monkey experiment) but onto the cortical surface reconstruction of the left and right hemisphere, respectively, of each monkey. We believe that this is an important methodological refinement for visualizing the Individual differences in the sulcal pattern. These differences are particularly remarkable in the posterior part of the brain (occipital, temporal and parietal cortices) where the main sulci can have considerably different shapes and sizes. Main sulci, as the parieto occipital sulcus (where macaque V6 is located), are different also in terms of their posterior-anterior extension. Moreover, the parieto occipital sulcus occupies a position which is slightly different also across the two hemispheres of a single animal. Rendering the individual results of each monkey on its surface would largely increase the chance to correctly assign the fMRI activity on the right side of the sulcus.

\section{Results}

\section{Whole-brain activations}

We first tested the responsiveness of macaque brain to low contrast radial motion (Radial Rings, Figure 1A), the stimulus used initially in humans to functionally map the motion middle temporal area MT/V5 (Tootell et al. 1995a,b) and later on routinely used also in our lab as MT+ localizer (e.g., Pitzalis et al., 2010, 2012, 2013a; Sulpizio et al., 2020).

Figure 2 shows results from Radial Rings motion experiment displayed on folded and inflated cortical surface reconstructions from the left and right hemispheres of the two monkeys. These functional maps show all the motion-sensitive regions showing a greater MION response (cluster-corrected $p<0.001$ ) to moving than to static Radial Rings in both monkey 1 (left) and monkey 2 (right).

This contrast revealed that preference for the radially moving stimuli defines a network of cortical regions spanning from the occipital, temporal to the parietal cortex. Specifically, one cortical activation site was found to prefer moving to static stimuli in both hemispheres of the two monkeys (four out of four hemispheres, $4 / 4 \mathrm{Hs}$ ). This region is located in the middle, superior portion of the superior temporal sulcus (STs) and likely includes several temporal motion regions known to be located in this part of the STs (see the following sections for details on the temporal activations). This site is the most activated functional spot we found in response to radial Ring stimulus in both animals, also surviving at very high thresholds $\left(p<10^{-10}\right)$.

Two cortical activation sites responded to Radial Rings in three out of four hemispheres (3/4 Hs). One site was found in the dorso-lateral subdivision of the Lunate sulcus in both hemispheres of $\mathrm{M} 1$ and in the right hemisphere of M2. This activation is large, including both the posterior bank (area V2 dorsal) and the fundus and anterior bank (areas V3 and V3A, respectively) of the Lunate sulcus. The second site was found in the fundus of the inferior occipital sulcus (IOs) in both hemispheres of $M 1$ and the right hemisphere of $M 2$. This site probably reflects area V2 ventral and might include VP.

Then we found two cortical activation sites in two out of four hemispheres $(2 / 4 \mathrm{Hs})$. Both sites were found along the intraparietal sulcus (both hemispheres of M1). One functional spot is located in the posterior end of the intraparietal sulcus (IPs) where the sulcus intersects the Lunate (LS) and the parieto occipital (POs) sulci. This is the typical position of CIP and PIP areas (likely including also the ventral part of the MIP area) and we refer to this region as pIPs (not visible here in the right hemisphere of Monkey 1 because of its 
posterior position, see flat maps of Figure 4). The other intraparietal spot is located more anteriorly in the middle portion of the IPs, facing the post-central gyrus and we refer to this region as dorsal Ips (dIPs). The location of this second spot might be consistent with the VIP location in the macaque.

Finally, we found three cortical activation sites in only one out of four hemispheres $(1 / 4 \mathrm{Hs})$. One functional spot is located on the very medial end of the Lunate sulcus at the intersection with the POs in the right hemisphere of M1. This position might correspond to the medial part of area V3A and may also reach the lateral part of $\mathrm{V} 6$ (see following sections for details). A second functional spot was found in the lateral extension of the occipital cortex (i.e., foveal representation of V1 and V2) in the left hemisphere of M2. The last site is located medially on the calcarine fissure (i.e., the periphery of V1 and V2) in the right hemisphere of M2. Note that this spot is somehow present also in the left and right hemispheres of M1 but with such a small size to be negligible (i.e., it doesn't reach an appreciable size in terms of number of voxels).

We then tested the responsiveness of the macaque brain to another type of motion stimulus, Flow Fields, which contrasts coherent optical flow stimulation with scrambled control (see Figure 1B). This stimulus was originally used in humans to functionally map the medial motion area V6 (Pitzalis et al. 2010) and later on routinely used in our lab as V6 localizer (e.g., Pitzalis et al., 2012, 2013a,c; Serra et al., 2019; Sulpizio et al., 2020).

Figure 3 shows results from Flow Fields experiment displayed on folded and inflated cortical surface reconstructions from the left and right hemispheres of the two monkeys. Results are rendered on both lateral and medial views. These functional maps show the motion-sensitive regions that showed a greater MION response $(p<0.001)$ to Flow Fields than to random motion in both tested monkeys. This contrast reveals that preference for the optic flow stimuli defines a more extensive network of cortical regions with respect to Radial Rings, located in both lateral and medial brain. These regions cover a large territory spanning from occipital, temporal, parietal, insular, precuneate, and cingulate cortices. Notably, activations in the temporal, medial parietal, and insular cortices were found very consistently across the two monkeys, while some other activated regions in the medial wall (precuneus and cingulate sulcus) and the Lunate sulcus were found less systematically. Specifically, we found five cortical activation sites in four out of four hemispheres (4/4 Hs). One site was found in the POs in a position that fits with the macaque area V6 (Galletti et al., 1996, 1999). Note that in humans, responses to optic flow have been found in both the medial parieto occipital areas V6 and V6Av (but not in V6Ad) and for this reason we typically refer to the Flow Field-related activation in the POs as V6 complex or V6+ (Pitzalis et al., 2006, 2013; Tosoni et al., 2015). Since here we don't have a retinotopic definition of macaque $V 6$, we cannot exclude that this functional spot includes also voxels from the neighboring anterior V6Av. For this reason, we refer to this region as POs or V6+.

Two functional spots were found along the STs. One is located in the middle, superior portion of the STs, extending up to the most dorso-caudal portion of the STs; the second STs spot is more ventrally located and is spatially separated from the more dorsal spot. Notice that this activation in the ventral STs was completely lacking in the Radial Rings activations. One further functional spot was found along the IPs, in correspondence of the pIPs region described above (not visible here in the left hemisphere of Monkey 2 because of its posterior position, see flat maps of Figure 4). Finally, one last functional spot was found in the insular cortex, immediately deep to the caudal portion of the lateral sulcus (Silvian fissure), facing the dorsal STs. We refer to this region as posterior insula (plns). We found one cortical activation site in the intraparietal sulcus in three out of four hemispheres $(3 / 4 \mathrm{Hs})$. This site was found in both hemispheres of $M 1$ and in the right hemisphere of M2. We refer to this region as dorsal intraparietal sulcus (dIPs). The site might correspond to the VIP region described above. We found two cortical activation sites in two out of four hemispheres (2/4 $\mathrm{Hs})$. One functional spot is located laterally in correspondence of the Lunate sulcus described above in both hemispheres of M2. The second functional spot is located medially in the anterior part of the precuneus and the caudalmost part of the cingulate sulcus in both hemispheres of M1. Likely, it involves areas PGm and 31 on the mesial surface, and area PEci in the caudal tip of the cingulate sulcus (Morecraft et al., 2004). Notably, while in the left hemisphere the cingulate activation could also involve the putative macaque CSv (pmCSv; Cotterau et al., 2017), in the right hemisphere it is not the case, the activation being clearly more posteriorly 
located. Finally, we found a cortical activation site in only one out of four hemispheres (1/4 Hs) in the left hemisphere of $\mathrm{M} 2$, located laterally in correspondence of the IOs regions described above.

Comparing Figures 2 and 3, it is evident that Flow Fields and Radial Rings do not activate the same portions of the STs. Although there is an overlapping area of activation, the overlap is only partial. To make a direct comparison of activation areas for Radial Rings and Flow Fields stimulations, we built up flat maps of the left and right hemispheres of the two studied monkeys where close-ups of the posterior part of the brain focused on the occipital and temporal regions and also included the very posterior parts of the IPs and Sylvian fissure (Figure 4). Figure $4 \mathrm{~A}$ shows that the greatest cluster of Radial Rings-related activation was found in the dorsal part of the superior temporal sulcus. As shown in Figure 4A, the portion of the STs activated by Radial Rings (mSTS) occupies a middle to superior location of the STs not reaching the very dorsal part of the sulcus. In absence of a retinotopic mapping or an independent a priori ROI definition, we hereafter refer to this cortical region as middle STS (mSTS). For ease of comparison, we traced a white border around the mSTS region obtained from Radial Rings experiment in both the hemispheres from the two monkeys and replicated these white contours on the Flow Fields maps shown in Figure 4B. This comparison shows that there is a region in the very dorsal part of the STs which is activated by Flow Fields but not by Radial Rings. We refer to this region as dorsal STS (dSTS). For reference, we traced a blue border around the dSTS region. In Flow Fields experiment but not in Radial Rings, we found a third functional spot in the ventral part of the STs which does not overlap with mSTS (Figure 4B). We refer to this region as anterior STS (aSTS).

The activation in the POs deserves some comment as well. In the macaque, the POs is a long sulcus dividing the occipital from the parietal lobe, that crosses the medial surfaces of the brain approaching ventrally the calcarine fissure. This sulcus presents a certain amount of individual variability in its shape and position. These individual differences in the anatomy are neglected when using anatomical templates, while they are totally revealed on 3D or 2D rendering of the real cortical surface of the animal. Observing the 3D surfaces shown in Figures 2 and 3 (where the POs is marked by a white dashed line), it is evident that the POs is nearly vertically oriented and slightly bent posteriorly in 3 out of four hemispheres (the right hemisphere of monkey 1 and the two hemispheres of monkey 2). In the left hemisphere of monkey 1 , on the contrary, the POs has bent anteriorly in its ventral part. In this hemisphere, the POs continues anteriorly into the medial parieto occipital sulcus (mPOs), on the mesial surface of the hemisphere. Note that in the right hemisphere of monkey 1 , the mPOs is clearly visible anterior to the POs but it is not confluent with POs itself. In monkey 2, the mPOs is present anterior to the POs but it is much less pronounced than in monkey 1 . The results of stimulus activations reported in Figure 2 show that Radial Rings does not activate the POs in both animals. An activation inside the POs is actually visible in the RH of M1, but Figure 4 shows that this activation is not medial enough to believe it involves area V6+. Possibly, it could involve the medial part of area V3. In contrast, Flow Fields stimulation does activate V6+ bilaterally in both M1 and M2 (Figures 3-4).

\section{Regional Analysis}

Beyond the whole-brain analysis, we further conducted a regional approach. While the whole-brain analysis was conducted to have a general picture of the brain regions involved in both Radial Rings and Flow Fields, the regional approach helps to distinguish regions responding only to Radial Rings or Flow Fields and those responding to both. Each ROI was first defined using only half of the runs at a relaxed threshold of clustercorrected $p<0.001$. Then, we extracted the average amplitude from each ROI using the other half of the runs (see the "Materials and Methods" section for details).

As stated in the Methods, in this regional approach, we focused our analysis on regions that were significantly activated in at least 3 out of the 4 hemispheres of our 2 monkeys. Following this inclusion criterion, we selected for further analysis the following nine ROIs: POs, dSTS, mSTS, aSTS, pIPs, dIPS, pIns, IOs, Lunate. In Figure 5, all ROIs are color-coded and are displayed together on the folded cortical surface reconstructions of the left hemisphere of monkey 1 (Figure 5A-B) and monkey 2 (Figure 5C). In Figure 5B, all ROls are also displayed on the inflated left hemisphere of monkey 1 , together with a nude version of the same hemisphere to indicate the relationship between the dorsal sulcal pattern and the ROI position. Figures 6-7 illustrate the functional response profiles of each $\mathrm{ROI}$ with respect to the two motion stimuli. The mean percentage signal 
changes we observed in Flow Fields (FF; Flow versus Random motion) and Radial Rings (RR; motion versus static conditions) are plotted (separately for each monkey and hemisphere) in the bar graphs of Figures 6-7. For each ROI, the two right-most bars refer to the average across monkey hemispheres for the two conditions. In the ROIs defined from Flow Fields (POs, dSTS, aSTS, pIns, dIPs, pIPs; Figure 6), we tested their response with respect to the other stimulus, Radial Rings, using one-sample $t$ tests versus zero in each region. Results revealed that two areas, dIPs and pIPs, significantly responded also to Radial Rings (dIPs, $t_{8}=9.11, p$ $=0.000021 ; \mathrm{pIPs}, \mathrm{t}_{8}=9.99, \mathrm{p}=0.000039$ ) while the other four areas, POs, dSTs, aSTs and plns did not (POs, $\mathrm{t}_{8}=1.47, \mathrm{p}=0.18 ; \mathrm{dST}, \mathrm{t}_{8}=2.82, \mathrm{p}=0.025 ; \mathrm{aSTs}, \mathrm{t}_{8}=-0.91, \mathrm{p}=0.39 ;$ ins, $\left.\mathrm{t}_{8}=1.37, \mathrm{p}=0.21\right)$. The same analysis was applied in the ROIs defined from Radial Rings (mSTs, IOs, Lunate; Figure 7) to test their response with respect to the other stimulus, Flow Fields. Results revealed that one area, mSTS, significantly responded also to Flow Fields (mSTs, $\mathrm{t}_{8}=9.83, \mathrm{p}=0.000024$ ) while the other two areas, IOs and Lunate, did not (IOs, $\mathrm{t}_{8}$ $=1.04, p=0.33$; Lunate, $t_{8}=1.05, p=0.32$ ). Overall, results indicate that three regions (mSTs, dIPs, pIPs) respond to both stimuli, four regions (POs, dSts, aSTs, pIns) respond only to Flow Fields and two regions (IOs, Lunate) respond only to Radial Rings. Any comparison between the differential sensitivity to Flow Fields and Radial Rings in each region is merely illustrative, due to the lack of independency between the data and the selection criteria.

Finally, in these regions, the specificity of the MION responses to FF versus RR stimuli was quantified by computing a sensitivity ratio (see the "Materials and Methods" section for details). Figure 8 shows these ratios separately for Flow Fields-defined ROls (Figure 8A) and Radial Rings-defined ROls (Figure 8B). A qualitative description of the results indicates that in Flow Fields-defined ROIs (Figure 8A) the preference of Flow Fields over Radial Rings is higher in POs, plns, dSTS and aSTS than in pIPs and dIPs (on average, about 4 times larger). In Radial Rings-defined ROIs (Figure 8B), the preference of Radial Rings over Flow Field is higher in IOs and Lunate than in mSTS (on average, about 2 times larger). Note that, in absence of an independent ROI definition, the sensitivity ratio was only aimed to qualitatively measure the strength of the relative effect among cortical region preferring Flow Fields or Radial Rings; it should not be meant to compare the differential sensitivity to Flow Fields and Radial Rings in each region.

\section{Discussion}

Cortical-surface-based functional MRI mapping techniques and motion stimulation were applied in two awake behaving macaques to describe the sensitivity of the monkey brain to two motion stimuli (Radial Rings and Flow Fields) originally used in humans to functionally map the motion middle temporal area MT+ (Tootell et al. 1995a,b) and the motion medial parietal area V6 (Pitzalis et al. 2010), respectively.

\section{Preference to Flow Fields in the parieto-occipital sulcus}

Our key finding concerns the activation found in the POs, that included area V6 and that here is called V6+. We wanted to test whether macaque V6+ was sensitive to Flow Fields like the human counterpart and whether this stimulus could be used as a functional localizer for macaque V6+, as it is for human V6+. Here we found a robust and consistent $\mathrm{fMRI}$ activations in $\mathrm{V} 6+$ for the contrast between Flow Fields and random motion (Figures 3-4). This region did not show any significant activation to Radial Rings stimulus, like the human counterpart (Pitzalis et al., 2010; Sulpizio et al., 2020). In addition, the average sensitivity ratio of POs (Figure $8 \mathrm{~A}$ ) indicates that $\mathrm{V} 6+$ was one of the motion areas with the greatest preference for the Flow Field with respect to the radial rings. In particular, the score of POs (0.8) means that in V6+ the Flow Field stimulus evoked about eight times the response of Radial Rings.

The retinotopic and functional properties of area V6 were originally described in macaque by the Galletti's group in the late nineties. Using simple visual stimuli like light/dark borders moved across the visual field, Galletti and coworkers (Galletti et al., 1996, 1999) found that the majority of V6 neurons were motion and direction sensitive, were very sensitive to the orientation of borders and responded well to large stimuli. Later, area V6 was identified also in humans by our group (Pitzalis 2006, 2010) and after that, a growing number of $\mathrm{fMRI}$ studies have described several functional properties of this area. Human V6 is specialized in distinguishing among different types of self-movement with a preference for the translational egomotion 
(Cardin and Smith 2010; Pitzalis et al. 2010, 2013b), and it is activated by the presence of stereoscopic depth cues associated with self-motion (Cardin and Smith 2011). Human V6 responds to changes of heading directions (Di Marco et al., 2021) and is thought to be involved in in the "flow parsing mechanism" contributing to perceptual stability during eye movements (Fischer et al., 2012a; Galletti et al., 1990; Galletti \& Fattori, 2003, 2018; Pitzalis et al. 2020). However, so far only few studies have tested the flow field sensitivity in macaque V6, and even with contrasting results. Fan and coworkers (2015), for instance, used a virtual reality system to examine the 3D heading tuning of macaque V6 neurons in response to either optic flow that simulated translational motion (visual condition) or actual platform motion (vestibular condition). They found similarities in the functional profile between V6, MSTd, and VIP, in that the majority of neurons in these regions were selective for heading defined by optic flow (in line with the results of the present study) and were able to discriminate small variations in heading around forward and backward headings. This last result is in line with a recent fMRI study from our group (Di Marco et al., 2021), where we compared the effect of a curved path (typically the visual feedback of a changing direction of movement in the environment) with respect to a linear path for simulated forward and backward motion. There we found that human V6+ (together with other regions like CSv and $\mathrm{pCi}$ ) have both a significant preference for the curved path motion and for the forward compared to backward motion. Fan et al. (2015) also found scarce evidence for vestibular heading signals in V6. Indeed, unlike other areas (as MSTd and VIP) V6 neurons were almost unresponsive to inertial motion in the absence of optic flow. These findings support the idea that $\mathrm{V} 6$ is involved primarily in processing visual motion signals at an early stage and does not appear to play a significant role in visualvestibular integration for self-motion perception.

While Fan et al. (2015) found that V6 cells showed robust responses to optic flow simulating translational motion, Cotterau et al. (2017) failed to find a preference of macaque V6 for egomotion compatible optic flow. A possible reason for this mismatch might be the type of visual stimulus used. The Flow Field stimulus used here and by Fan et al. (2015) produces a pattern of coherent motion stimulation similar to the continuously changing optic flow generated when a person moves through in a complex environment (Koenderink 1986). Cardin and Smith (2010), instead, reported that participants did not experience vection. Unlike the egomotion consistent stimulus used by Cardin and Smith (2010), Flow Fields is very powerful in inducing in human subjects a compelling perception of self-motion (vection) and this could be a window into the function of V6, that is supposed to be involved in the analysis of visual signals during locomotion (e.g., Di Marco et al., 2021). Another possible explanation for the mismatch is that in the stimulus used by Cotterau, coherent motion, although at different extent, is present in both the egomotion-consistent (EC) and egomotion-inconsistent (EI) blocks. If this is the case, the local motion coherence could excite $\mathrm{V} 6$ cells during both blocks, masking the selectivity to EC coherent motion. Finally, it could be possible that the continuous and rapid visual changes occurring on the retina during the Flow Fields stimulation is particularly effective in activating $\mathrm{V} 6+$. It might be that, because of the strict direction and orientation selectivity of V6 cells (Galletti et al. 1996), the total number of cells activated by a single moving step in the stimulus by Cardin and Smith (2010) is much less than the total number of cells activated by the flow field stimulus, where direction and speed of movement, as well as the type of movement coherence, changed every $500 \mathrm{~ms}$.

In summary, here we used the same stimuli previously used to map human V 6 and our results show that a macaque region within POs, likely including area V6, strongly responded to Flow Fields, like human V6. This similarity in the pattern of response across both species suggests that these cortical areas in humans and macaques might be homologous. It remains for additional studies to clarify whether $\mathrm{V} 6$ is involved in heading perception and/or it play a role in flow parsing operations (Rushton and Warren, 2005; Warren and Rushton, 2008,2009 ) to dissociate object-motion and self-motion as suggested by several authors so far (Fan et al., 2015, Pitzalis et al., 2010, 2013, Galletti and Fattori, 2003, 2018).

\section{Preference to Flow Fields in other regions}

Another aim of the present study was to verify whether the Flow Field stimulus, routinely used in humans to map a set of cortical egomotion areas (Pitzalis et al., 2010; Serra et al., 2019), could be effective also on macaque brain.

In humans, we recently showed that at single-subject level, the Flow Field stimulus activated a network of six egomotion-selective areas (V6+, V3A, IPsmot/VIP, pCi, PIC, and CSv; see Serra et al. 2019) similar to that identified by Smith and coworkers using an egomotion-consistent/inconsistent stimulation 
(Wall et al. 2008; Cardin and Smith, 2010). In Serra et al. (2019) we found that the Flow Field stimulus activates also other regions in the human temporal cortex (dorsal portion of the STs and MT+) although less consistently with respect to the other regions. Here, we report similar results on monkey brain. Indeed, to areas in the POs (including V6 and already discussed in the previous paragraph), we found that Flow Fields activate also a set of motion areas in parietal (dIPS, pIPs), temporal (dSTS, aSTS) and insular (plns) cortices. Some of these areas (aSTS, dSTS, pIns) are exclusively activated by the flow field stimulus compared to Radial Rings. Other regions show a significant response also to Radial Rings (dIPS, pIPs). The mean sensitivity ratio of these regions (Figure $8 \mathrm{~A}$ ) indicates that the relative effect size in dSTS, aSTS and plns was as high as that in V6+, suggesting a strong preference in all these areas for Flow Fields. The flow field preference in dSTS, aSTS and plns is discussed in more detail in the following paragraphs.

Activations in Temporal regions (dSTS, aSTS).

Here we found significant fMRI activations in dSTs for the contrast between Flow Fields and random motion (Figures 3-4). Area dSTs is an egomotion selective region responding only to Flow Fields (Figure 6), with an average sensitivity ratio comparable (only slightly lower) to that in the POs (Figure 8A). The optic flow sensitivity we found in the dorsal part of the STS was already reported in the past macaque literature. Indeed, dSTS as defined here might correspond to the dorsal medial superior temporal (MSTd) area, a macaque region known for being sensitive to optic flow and to egomotion related signals (Nelissen et al., 2006; Saito et al. 1986; Tanaka et al., 1986, 1989; Tanaka and Saito, 1989; Lagae et al., 1994; Siegel and Read, 1997; Oram and Perrett, 1994; Duffy and Wurtz, 1991a,b; Duffy, 1998; Orban et al. 1992; Graziano et al. 1994; Eifuku and Wurtz, 1998; De Angelis et al., 2012; Cotterau et al., 2017). Cotterau et al. (2017) found that in MSTd the sensitivity ratio between responses to egomotion consistent (EC) and egomotion inconsistent (EI) flow stimuli was moderate (although higher than in humans; Wall and Smith 2008), suggesting that also El can evoke strong responses in MSTd neurons. Interestingly, in macaque, MST neurons present response properties similar to those of $\mathrm{V} 6$ neurons, in that both have large receptive fields, responsive to direction and speed of moving visual stimuli and both receive strong direct inputs from V1 (Boussaoud et al. 1990; Sousa et al. 1991; Palmer and Rosa 2006; Galletti et al. 2001). These two areas are also directly interconnected (Galletti et al. 2001). Given these similarities, it is possible that the same neuronal mechanisms can be raised to explain their common functional responsiveness to key features of flow field and that these two areas share many functional properties.

Also in human, several past studies led to a general agreement in reporting MSTd as an area sensitive to the motion coherence and to egomotion signals (Morrone et al. 2000; Kleinschmidt et al. 2002; Smith et al. 2006; Wall et al. 2008; Kovacs et al. 2008; Helfrich et al. 2013; Arnoldussen et al., 2011; Pitzalis et al., 2013, 2020). Notably, the high and comparable preference for Flow Field observed here in V6+ and dSTS (Figure $8 \mathrm{~A}$ ) does not resemble the trend observed in humans, where our (Pitzalis et al., 2013c, 2020) and other (Kleinschmidt et al. 2002; Wall and Smith, 2008) labs found a moderate MST preference for the self-motion compared to that observed in other areas, like V6 and CSV, meaning that human MST responds to egomotion but is not strongly selective for it. It is also possible that the dSTS as defined here includes voxels from neighboring motion regions. Future studies using strict definition criteria are needed to definitively clarify this point.

The optic flow preference found here in aSTs, in correspondence of the most anterior portion of the STs, is in line with previous single-cell studies in monkey showing neurons selective to optic flow in the anterior portion of the STS (Bruce et al. 1981; Anderson and Siegel 1999; Nelissen et al., 2006). Notably, we found fMRI activity mainly in correspondence of the upper bank and fundus of the STs, where neurophysiological studies have described the location of the superior temporal polysensory (STP) area. Although the function of the STP region remains mostly unclear, it seems that at least part of this region is involved in the analysis of optic flow (e.g., Bruce et al., 1981; Anderson and Siegel, 1999). Indeed, Anderson and Siegel (1999) found that neurons within the anterior STP (STPa) are motion selective areas responding to the translation, rotation, radial, and spiral optic flow components and contributing to the analysis of optic flow likely with the aim of guiding forward locomotion. Optic flow sensitivity was also found in correspondence with the middle part of the STP, called STPm (Nelissen et al., 2006; Cotterau et al., 2017). In particular, Nelissen et al. (2006) found that neurons within the STPm are sensitive to optic flow stimuli and are likely involved in the visual analysis of actions and biological motion. However, the ventral activation 
found here in Flow Fields experiments seems more anterior than the STPm (Nelissen et al., 2006) and more likely corresponding to the STPa reported by Anderson and Siegel (1999). Future studies using an independent ROI definition are needed to establish the correspondence between the region found here and the previous literature.

The human counterpart of macaque STPa could be the STSms (STS multi-sensory) area, a multisensory region found anteriorly to MT+, responding to visual (Beauchamp et al. 2004a, 2004b, 2008) and to vestibular (Smith et al. 2012) signals as well. Note however that in humans, the STSms is not significantly activated by our Flow Fields stimulus (Pitzalis et al., 2010; Serra et al., 2019) nor by Cardin and Smith (2010) egomotion compatible/incompatible stimulus. Thus, the high sensitivity to optic flow of the anterior portion of the macaque superior temporal sulcus suggests the possibility of a specialization of that part of the monkey brain for the egomotion perception which does not have a strong counterpart in humans.

Activations in the vestibular Cortex (plns). An egomotion-related activation was found in the posterior insular region, in the caudal portion of the sylvian fissure (see Figure 5). The location of this activation site might correspond to the visual posterior Sylvian (VPS) area that has been recently shown to process optic flow in the macaque (Chen et al., 2011a,b; Cotterau et al., 2017). Human studies have demonstrated that even only the passive viewing of optic flow stimuli is effective in reliably localizing the human homologous of the macaque VPS (called in human PIC, parieto insular cortex region; Claeys et al., 2003; Cardin and Smith, 2010; Chen et al., 2011a,b; Frank et al. 2014; Serra et al., 2019: Pitzalis et al., 2020). Present and previous (Cotterau et al., 2017) fMRI results show that the Flow Field stimulus can be used to activate part of the vestibular network also in the macaque. Note that the high preference for Flow Fields found here in plns was comparable to that observed in V6+ and in the temporal regions (dSTS and aSTS) (Figure 8A). This is in line with previous report (Cotterau et al., 2017) where the authors found in this region one of the highest sensitivity ratio value (computed between EC and EI). We missed observing fMRI activation in the vestibular area 2v (putative 2v or p2v; Guldin and Grusser, 1998; Cardin and Smith, 2010). Note however that both monkey (Cotterau et al., 2017) and human studies (Serra et al., 2019) recently failed to find this region activated in response to optic flow stimuli.

Activations in the Cingulate Cortex. Direct comparison of present results from Flow Field experiment with the equivalent human study (Pitzalis et al., 2010) reveals several commonalities but also some differences. One of the most evident difference is about the functional properties of cingulate area CSv, first found in humans by Wall and Smith (2008) and recently described also in macaque (Cotterau et al., 2017). Many fMRI studies found that human CSv is sensitive to wide-field egomotion-compatible stimuli (Antal et al., 2008; Cardin and Smith. 2010; Fischer et al., 2012a; Pitzalis et al., 2013c; Field et al., 2015; Wada et al., 2016), and the recent study by Cotterau et al. (2017) reported the same sensitivity for macaque CSv. However, human CSv has been repeatedly found in fMRI experiments using visual motion stimuli, whereas macaque CSv was rarely found in such a kind of experiments. Here we did not find clear evidence of a reliable response to Flow Fields in macaque CSv, and also Cotterau et al. (2017) found a moderate involvement of CSv in the egomotion perception, and in any case much less robust responses than those observed in the same study for other areas (like MSTd, VIP, VPS). The conclusion is that more studies are needed to identify a proper stimulus to activate this region in the monkey brain.

We missed observing $\mathrm{fMRI}$ activation also in a cingulate region which is commonly activated in $\mathrm{fMRI}$ studies using similar (or identical) stimuli, like the cingulate area pC (Cardin and Smith, 2010), later on renamed PcM (Cardin and Smith, 2011) or pCi (Serra et al., 2019). In the monkey, also Cotterau et al. (2017) did not find egomotion-related activation in this region of the brain.

\section{Preference to Radial Rings in mSTS, striate and extrastriate occipital areas}

Direct comparison of the present results on Radial Rings experiment with the equivalent human study (Pitzalis et al., 2010) reveals striking commonalities. Indeed, as in humans, here we report that this stimulus elicited a strong functional activation in the middle superior temporal sulcus, in the mSTS region, consistently across the two monkeys ( $4 / 4$ hemispheres). Yet as in humans, this stimulus consistently activated also 
extrastriate dorsal occipital regions located in the Lunate sulcus, likely corresponding to areas V2d, V3, and V3A. The Radial Rings preference in these regions is discussed in more detail in the following paragraphs.

Here, we found a robust and consistent fMRI activations in $\mathrm{mST}$ s for the contrast between Radial motion and static rings (Figures 2, 4). The response in this region was not selective for Radial Rings, in that in the regional analysis mSTs also showed a significant activation to Flow Fields stimulus (Figure 7). In addition, although the average sensitivity ratio in $\mathrm{mSTs}$ (Figure 8B) is coherent with a preference for Radial Rings (being the score smaller than zero), it is much lower than that observed in Lunate and IOs. This indicates that mSTs has a reliable and statistically significant response to both Radial Rings and Flow Fields. The anatomical location and functional profile of $\mathrm{mSTs}$ is in line with macaque data on motion area MT+. Indeed, MT neurons respond equally well to a bar as to random dot patterns in translation (Tanaka et al., 1986), and MT neurons respond as well to optic flow fields as to translating random dots (Lagae et al 1994; Nelissen et al 2006). The functional profile observed here in mSTs also mirrors that observed in the human counterpart, where MT+ (identified by Radial Rings) was found to respond, although at different extent, also to Flow Fields besides to Radial Rings (Sulpizio et al., 2020).

The Radial Rings preference found in the mSTs supports the presence of different functional profiles in neighboring regions along the STS, in line with previous fMRI studies on monkey (e.g., Vanduffel et al., 2014; Cotterau et al., 2017; Hejja-Brichard et al., 2020). Indeed, as reported above, while dSTs and aSTs responds only to Flow Fields, mSTs responds to both Flow Fields and Radial Rings. In addition, the Flow Field preference score in mSTS was opposite to that in dSTS and aSTS (Figure 6): while dSTs and aSTS were preferentially activated in the Flow Field experiment, mSTS was preferentially activated in Radial Rings experiment. These results point to a different functional profile of these temporal regions despite their vicinity. We are aware that the ROIs definition used here sets the border between these three regions quite arbitrarily (since it is affected by the chosen statistical threshold), but it still has the advantage to allow us to determine whether the functionally selected territory showed different functional properties despite their vicinity to each other.

Several previous macaque neural recording studies have described a number of motion-sensitive regions within the STS: the MT cluster or MT+; Kolster et al 2009), that includes besides the well-known MT/V5 other neighboring areas, such as FST ventrally (Gattass and Gross 1981; Van Essen et al. 1981; Desimone and Ungerleider 1986; Sereno et al. 1994), VMST (Tanaka et al. 1993) and V4T posteriorly (Desimone and Ungerleider 1986; Gattass et al. 1988). Nelissen et al. (2006) found a new motion sensitive region on the lower bank and fundus of the STS, anterior to the FST area, termed LST (lower superior temporal). This region responds to slow as well as fast speeds and is responsive to static and moving images of objects. Since we don't have a retinotopic mapping of the region, we cannot unambiguously settle the correspondence between mSTS, as defined here, and other functional temporal regions. However, the anatomical position of mSTS suggests it may overlap either the MT+ cluster or dorsally the LST described by Nelissen et al (2006). Notably, LST region was found to respond to moving random dots and also more to moving than to static bars (Nelissen et al 2006), a functional profile mirroring that observed here for mSTS.

The motion sensitivity observed here in the anterior bank of the Lunate sulcus, likely corresponding to macaque V3A, deserves a comment. Since its discovery in humans (Tootell et al., 1997), a growing number of $\mathrm{fMRI}$ studies have reported that human V3A is significantly activated by coherent motion and by complex motion patterns as the Flow Field stimulus (e.g., Sunaert et al., 1999; Sereno et al., 2001; Pitzalis et al., 2010, 2013c; Fischer et al., 2012b; Serra et al., 2019; Sulpizio et al., 2020). In contrast, the motion sensitivity of macaque V3A is still a debated question. Several studies have shown that the same motion stimulation able to activate V3A in human was not effective in activating V3A in macaque (Orban et al., 2003; Abdollahi et al., 2014). However, it was demonstrated that V $3 A$ single cells in macaque are strongly activated by moving stimuli, several of them are direction selective and most of them are activated by the real motion of an object, but not by retinal image motion of the same object self-evoked by eye movements (real-motion cells; Galletti et al 1990). Very recently, Nakhla et al. (2021) have reported data in support to the view that macaque V3A bears characteristics of a "motion area". These authors reported that direction selectivity of single V3A cells in macaque was high and similar in V3A and MT, and that V3A neurons exhibits somewhat greater selectivity for optic flow patterns than that in MT. Future experiments, using a retinotopic mapping to define V3A will certainly add further material to this debate. 


\section{Conclusive remarks}

A final note goes to the comparison between the two most important motion centers of the dorsal stream in both human and macaque: the POs on the medial wall and the STS on the lateral surface.

Here we found a different functional profile for these two motion centers when optic flow or radial motion stimuli are used. The POs (likely including V6) is well activated by the coherent motion of dot fields (Flow Fields) but not by radial motion (Radial Rings), as in humans (Pitzalis et al. 2010; Sulpizio et al., 2020). Conversely, the mSTS in the temporal cortex (likely including MT+) is well activated by Radial Rings (the stimulus classically used to map human MT+; Tootell et al., 1995a,b) and also by Flow Fields, although at a lower extent. The mSTS profile found here parallels that observed in human MT+. While in a first paper, we reported that the Flow Field stimulus did not elicit statistically significant responses in MT+ (see e.g. Pitzalis et al. 2010), in more recent papers we have found that MT+ shows some degree of preference for egomotioncompatible optic flow, although with a weak consistency across subjects (lower than 70\%; Serra et al. 2019; Sulpizio et al., 2020). Also, Cardin and Smith (2010) found significant responses to egomotion-compatible optic flow in MT+, although selectivity in this area was not as pronounced as in VIP and CSv (Wall and Smith 2008). Present results on monkey brain go along the same direction confirming our recent observations on humans of a significant response in the mSTS (likely including MT+) for both Radial Rings and Flow Field stimulus.

The above reported results suggest that the two dorsal motion centers (V6+, MT+) perform different functions. Many years ago, it was suggested (Previc, 1998; Previc et al., 2000; Rosa \& Tweedale, 2001) that lateral (MT+) and medial ( $\mathrm{V}^{+}+$) motion areas are engaged in the detection of object-motion and self-motion, respectively. In accordance with this hypothesis, in a recent fMRI humans study testing self- and objectmotion in a naturalistic vision (Pitzalis et al., 2020), we have found a lateral area (MT+) was particularly sensitive to object-motion and several medial motion areas ( $\mathrm{PEc}, \mathrm{pCi}, \mathrm{CSv}$, and $\mathrm{CMA}$ ) preferred self-motion. However, we failed to find a strict segregation of functions, since the medial motion area V6+ responded equally well to both self- and object-motion, and some motion areas of the lateral surface (PIC and LOR) preferred self-motion. Galletti and Fattori $(2003,2018)$ suggested an alternative hypothesis. According to these authors, MT/V5 would be involved in the analysis of motion signals (direction and speed of movement) particularly in the central part of the visual field, whereas V6 would be involved in "subtracting out" selfmotion across the whole visual field. The fact that the representation of the periphery in V6 is more emphasized in comparison with that in area MT/V5 (Gattass and Gross 1981; Galletti et al. 1999; Pitzalis et al. 2006) lends support to this view. Also in humans, area V6 is an egomotion-related region with properties that constitute important prerequisites for a 'visual analysis' of the retinal signals evoked by self-motion. First, V6 is retinotopically organized and respond to the entire contralateral hemifield up to the very far periphery (Pitzalis et al 2006). Second, it is well activated by optic flow (Cardin and Smith, 2010: Cardin et al., 2012a; Morrone et al., 2000; Wall and Smith, 2008; Pitzalis et al 2010, 2012, 2013b, 2015; Serra et al., 2019), which constitutes a rich source of visual cues that can facilitate navigation through the environment. Third, V6 is specialized in distinguishing among different types of self-movement, showing a strong response to translational egomotion (Pitzalis et al., 2013c) that allows extracting information about the relative distance of objects, useful to act on them or to avoid them (see also Cardin et al., 2012b). Fourth, V6 prefer complex visual stimulation where both self- and object-motion are present in the stimulus, similarly to what happens in daily life when people move in a dynamic and complex environment (Pitzalis et al., 2020).

To summarize, area V6 could be involved in the perceptual stability despite eye movements (Galletti and Fattori 2003, 2018; Fischer et al., 2012b) and could contribute to distinguish object motion from the retinal image motion evoked by self-motion (Warren \& Rushton, 2009a, 2009b; Galletti and Fattori 2018), particularly when the subject moves through complex environment. Future human and macaque fMRI studies playing off central and peripheral cues to self-motion and object motion will verify the validity of these hypotheses. 


\section{Legends}

Figure 1. Experimental paradigm. The panels represent the two block-design experiments (Radial Rings and Flow Fields) performed separately in different fMRI runs and sessions. A. The upper panel shows the Radial Rings stimuli. The ON phase consisted of expanding and contracting rings alternating at a frequency of 0.5 $\mathrm{Hz}$. The OFF phase consisted of stationary rings. B. The lower panel shows the Flow Fields stimuli. The ON phase consisted of coherent radial and rotation-spiral motion alternating at a frequency of $2 \mathrm{~Hz}$. The OFF phase consisted of random motion. For all stimuli, we used a standard stimulation $\left(36^{\circ}\right.$ wide of visual field tested) and the animals were trained to maintain steady fixation on the central cross to minimize eye movements. See Materials and Methods for further details.

Figure 2. Whole brain analysis. Individual fMRI activations for the Radial Rings stimulus superimposed on left and right folded and inflated cortical surface reconstructions of the left and right hemispheres of Monkey 1 (left) and Monkey 2 (right). Results are rendered on both lateral and medial views. Figure shows the differentiated MION activity between ON and OFF conditions. Red regions indicate higher MION activity (cluster-corrected $\mathrm{p}<0.001$ ) during radially moving rings than during stationary patterns. In inset, an example of the Radial Rings stimulus we used. Major sulci (dark gray) are labeled as follows: as, arcuate sulcus; CS, central sulcus; IPs, intraparietal sulcus; syf, sylvian fissure; STs, superior temporal sulcus; Ls, Lunate sulcus; IOs, inferior occipital sulcus; Cgs, cingulate sulcus; POs, parieto occipital sulcus; calc, calcarine.

Figure 3. Whole brain analysis. Individual fMRI activations for the Flow Fields stimulus superimposed on left and right folded and inflated cortical surface reconstructions of the left and right hemispheres of Monkey 1 (left) and Monkey 2 (right). Results are rendered on both lateral and medial views. Figure shows the differentiated MION activity between ON and OFF conditions. Red regions indicate higher MION activity (cluster-corrected $\mathrm{p}<0.001$ ) during coherent random dot fields (outward and inward radial and spiral motion) than during scrambled moving random dot fields. In inset, an example of the Flow Fields stimulus. Main sulci have text label as in Figure 2.

Figure 4. Whole brain analysis. Individual fMRI activations for the Radial Rings (A) and Flow Fields (B) stimuli superimposed on flat maps of the left and right hemispheres of Monkey 1 (left) and Monkey 2 (right). In inset, an example of both the Radial Rings and Flow Fields stimuli. Major sulci (dark gray) have text label as in Figures 2 and 3.

Figure 5. Brain location of the Regions of Interest (ROIs). A. A folded (lateral, medial and superior views) representation of the left hemisphere (LH) of Monkey 1 marked with the locations of nine ROIs: dIPs (dorsal IntraParietal sulcus), pIns (posterior Insula), dSTS (dorsal Superior Temporal Sulcus), mSTs (middle Superior Temporal sulcus), aSTs (anterior Superior Temporal sulcus), pIPs (posterior Intraparietal sulcus), Lunate, IOs (Inferior Occipital sulcus), POs (Parieto Occipital sulcus). B. An inflated representation (superior views) of the LH of the same Monkey 1. Figure shows also a 'naked' version of the inflated hemisphere to indicate the relationship between the dorsal sulcal pattern and the ROI position. The dashed lines reported on the inflated representations indicate the fundus of the major sulci (dark gray) labeled as follows: As, anterior sulcus; CS, central sulcus; IPs, intraparietal sulcus; Sfy, sylvian fissure; STs, Superior Temporal sulcus; Ls, Lunate sulcus; IOs, inferior occipital sulcus; POs, parieto occipital sulcus; Cgs, cingulate sulcus. C. Folded (lateral, medial and superior views) representation of the LH of Monkey 2 marked with the locations of eight out of nine ROls. Other labels are as in Figure 5A-B.

Figure 6. Regional analyses. Functional response profiles of the six ROls defined using Flow Fields (POs, pIPs, dIPs, dSTs, aSTs, pIns). Plots for each region represent the averaged MION percent signal change \pm standard error of the mean for Flow Fields (FF; Flow versus Random motion) and Radial Rings (RR; motion versus static conditions), plotted separately for each monkey and hemispheres. For each ROI, the two right-most bars refer to the average (Avg) across monkey hemispheres for the two tasks. ${ }^{*} p<0.0001$ (Bonferroni corrected).

Figure 7. Regional analyses. Functional response profiles of the three ROIs defined using Radial Rings (mSTs, IOs, Lunate). The plots for each region represent the averaged MION percent signal change \pm standard error 
of the mean for Flow Fields (FF; Flow versus Random motion) and Radial Rings (RR; motion versus static conditions), plotted separately for each monkey and hemispheres. For each ROI, the two right-most bars refer to the average (Avg) across monkey hemispheres for the two tasks. ${ }^{*} p<0.0001$ (Bonferroni corrected).

Figure 8. Sensitivity ratio. Specificity of the MION responses to FF versus RR stimuli (FF - RR)/ (FF + RR) computed for (A) the six ROIs defined using Flow Fields (POs, pIPs, dIPs, dSTs, aSTs, plns) and (B) the three ROls defined using Radial Rings (mSTs, IOs, Lunate). Scores equal to zero imply same activation for Flow Fields and Radial Rings, scores greater than zero imply higher activation for Flow Fields than Radial Rings, while scores smaller than zero imply higher activation for Radial Rings than Flow Fields (see Methods).

Tab 1. This table lists all the acronyms used in the paper to indicate macaque brain surface Regions of Interest (ROIs).

\section{Declaration of Competing Interest}

The authors declare that they have no known competing financial interests or personal relationships that could have appeared to influence the work reported in this paper.

\section{Funding}

The work was supported by the University of Foro Italico, Rome, grant to S.P. (CDR2.FFABR). 


\section{References}

Abdollahi R.O., Kolster H., Glasser M.F., Robinson E.C., Coalson T.S., Dierker D., Jenkinson M., Van Essen D., Orban G.A. (2014). Correspondences Between Retinotopic Areas and Myelin Maps in Human Visual Cortex. Neuroimage, 99(100): 509-24.

Anderson KC, Siegel RM. (1999). Optic flow selectivity in the anterior superior temporal polysensory area, STPa, of the behaving monkey. J Neurosci. 19:2681-2692

Antal A, Baudewig J, Paulus W, Dechent P (2008). The posterior cingulate cortex and planum temporale/parietal operculum are activated by coherent visual motion. Vis Neurosci 25(01): 17-26.

Arnoldussen DM, Goossens J, van den Berg AV (2011). Adjacent visual representations of self-motion in different reference frames Proc Natl Acad Sci USA 108(28), 11668-73 doi: 101073/pnas1102984108

Beauchamp MS, Argall BD, Bodurka J, Duyn JH, Martin A. (2004a). Unraveling multisensory integration: patchy organization within human STS multisensory cortex. Nat Neurosci. 7: 1190-1192.

Beauchamp MS, Lee KE, Argall BD, Martin A. (2004b). Integration of auditory and visual information about objects in superior temporal sulcus. Neuron. 41:809-823

Beauchamp MS, Yasar NE, Frye RE, Ro T. (2008). Touch, sound and vision in human superior temporal sulcus. Neuroimage. 41:1011-1020.

Bremmer F, Schlack A, Shah NJ, Zafiris O, Kubischik M, Hoffmann K, Zilles K, Fink GR (2001). Polymodal Motion Processing in Posterior Parietal and Premotor Cortex: a human fMRI study strongly implies equivalencies between humans and monkeys. Neuron 29(1): 287-296.

Bruce C, Desimone R, Gross CG. (1981). Visual properties of neurons in a polysensory area in superior temporal sulcus of the macaque. J Neurophysiol. 46:369-384.

Cardin V, Hemsworth L, Smith AT (2012b). Adaptation to heading direction dissociates the roles of human MST and V6 in the processing of optic flow. J Neurophysiol 108(3): 794-801.

Cardin V, Sherrington R, Hemsworth L, Smith AT (2012a). Human V6: functional characterisation and localization. PLoS One 7(10): e47685.

Cardin V, Smith AT (2010). Sensitivity of human visual and vestibular cortical regions to egomotioncompatible visual stimulation. Cereb Cortex 20 (8): 1964-73.

Cardin V, Smith AT (2011). Sensitivity of human visual cortical area V6 to stereoscopic depth gradients associated with self-motion. J Neurophysiol 106: 1240-1249.

Chen A, DeAngelis GC, Angelaki DE. (2011a). Representation of vestibular and visual cues to self-motion in ventral intraparietal cortex. J Neurosci. 31:12036-12052.

Chen A, DeAngelis GC, Angelaki DE. (2011b). Convergence of vestibular and visual self-motion signals in an area of the posterior sylvian fissure. J Neurosci. 31:11617-11627.

Cottereau BR, Smith AT, Rima S, Fize D, Héjja-Brichard Y, Renaud L, Lejards C, Vayssière N, Trotter Y, Durand JB. (2017). Processing of Egomotion-Consistent Optic Flow in the Rhesus Macaque Cortex. Cereb Cortex. 2017 Jan 1;27(1):330-343. doi: 10.1093/cercor/bhw412.

Cox R.W. (1996). AFNI: Software for Analysis and Visualization of Functional Magnetic Resonance Neuroimages. Comput Biomed Res, 29(3): 162-73.

Dale AM, Fischl B, Sereno MI (1999). Cortical surface-based analysis I Segmentation and surface reconstruction. Neuroimage 9: 179-194.

DeAngelis GC, Angelaki DE. Visual-Vestibular Integration for Self-Motion Perception. In: The Neural Bases of Multisensory Processes. CRC Press/Taylor \& Francis, Boca Raton (FL); 2012. 
Desimone R, Ungerleider LG. (1986). Multiple visual areas in the caudal superior temporal sulcus of the macaque. J Comp Neurol. 248:164-189.

Duffy CJ (1998). MST neurons respond to optic flow and translational movement. J Neurophysiol 80(4): 181627.

Duffy CJ, Wurtz RH. (1991a). Sensitivity of MST neurons to optic flow stimuli. I. A continuum of response selectivity to large-field stimuli. J Neurophysiol. 65(6):1329--1345.

Duffy CJ, Wurtz RH. (1991b). Sensitivity of MST neurons to optic flow stimuli. II. Mechanisms of response selectivity revealed by small-field stimuli. J Neurophysiol. 65(6):1346--1359.

Duhamel JR, Colby CL, Goldberg ME (1998). Ventral intraparietal area of the macaque: congruent visual and somatic response properties. J Neurophysiol 79(1): 126-36.

Dupont P, De Bruyn B, Vandenberghe R, Rosier AM, Michiels J, Marchal G, Mortelmans L, Orban GA (1997). The kinetic occipital region in human visual cortex. Cereb Cortex 7(3): 283-92.

Eifuku S, Wurtz RH, (1998). Response to motion in extrastriate area MSTI: center-surround interactions. J Neurophysiol 80(1): 282-96.

Fan RH, Liu S, DeAngelis GC, Angelaki DE. (2015). Heading tuning in Macaque Area V6. J Neurosci. 35:1630316314.

Field DT, Inman LA, Li L (2015). Visual processing of optic flow and motor control in the human posterior cingulate sulcus. Cortex 71: 377-89.

Fischer E, Bülthoff HH, Logothetis NK, Bartels A (2012a). Visual motion responses in the posterior cingulate sulcus: a comparison to V5/MT and MST. Cereb Cortex 22(4): 865-76.

Fischer E, Bülthoff HH, Logothetis NK, Bartels A. (2012b). Human areas V3A and V6 compensate for selfinduced planar visual motion. Neuron. Mar 22;73(6):1228-40. doi: 10.1016/j.neuron.2012.01.022.

Fischl B, Sereno MI, Dale AM (1999). Cortical surface-based analysis: II: inflation, flattening, and a surfacebased coordinate system. Neuroimage, 9: 195-207.

Frank SM, Baumann O, Mattingley JB, Greenlee MW (2014). Vestibular and visual responses in human posterior insular cortex. J Neurophysiol 112(10): 2481-91.

Furlan, M., Wann, J. P., \& Smith, A. T. (2014). A representation of changing heading direction in human cortical areas pVIP and CSv. Cerebral Cortex, 24(11), 2848-2858. http://doi.org/10.1093/cercor/bht132

Galletti C and Fattori P (2018). The dorsal visual stream revisited: stable circuits or dynamic pathways? Cortex In press DOI: 101016/jcortex201701009.

Galletti C, Battaglini PP, Fattori P (1990). 'Real-motion' cells in area V3A of macaque visual cortex. Exp Brain Res 82: 67-76.

Galletti C, Fattori P (2003). Neuronal mechanisms for detection of motion in the field of view. Neuropsychologia 41: 1717-1727.

Galletti C, Fattori P, Battaglini PP, Shipp S, Zeki S. (1996). Functional demarcation of a border between areas V6 and V6A in the superior parietal gyrus of the macaque monkey. Eur J Neurosci. 8:30--52.

Galletti C, Fattori P, Gamberini M, Kutz DF. (1999). The cortical visual area V6: brain location and visual topography. Eur J Neurosci. 11: 3922--3936.

Gattass R, Gross CG. (1981). Visual topography of striate projection zone (MT) in posterior superior temporal sulcus of the macaque. J Neurophysiol. 46:621-638. 
Gattass R, Sousa APB, Gross CG. (1988). Visuotopic organization and extent of V3 and V4 of the macaque. J Neurosci. 8:1831-1845.

Georgieva SS, Todd JT, Peeters R, Orban GA (2008). The extraction of 3D shape from texture and shading in the human brain. Cereb Cortex 18(10): 2416-2438.

Graziano, MS, Andersen, RA, Snowden, RJ (1994). Tuning of MST neurons to spiral motions. J Neurosci 14(1): 54-67.

Greenlee MW, Frank SM, Kaliuzhna M, Blanke O, Bremmer F, Churan J, Cuturi LF, MacNeilage PR, Smith AT (2016). Multisensory Integration in Self Motion Perception. Multisens Res, 29 (6-7): 525-556.

Guldin WO, Grüsser OJ (1998). Is there a vestibular cortex? Trends Neurosci 21: 254-259.

Hadj-Bouziane F, Bell AH, Knusten TA, Ungerleider LG, Tootell RB (2008). Perception of emotional expressions is independent of face selectivity in monkey inferior temporal cortex. Proc Natl Acad Sci USA 105(14):5591-5596.

Hadj-Bouziane F, Liu N, Bell AH, Gothard KM, Luh WM, Tootell RB, Murray EA, Ungerleider LG. (2012). Amygdala lesions disrupt modulation of functional MRI activity evoked by facial expression in the monkey inferior temporal cortex. Proc Natl Acad Sci USA. Dec 26;109(52):E3640-8. doi: 10.1073/pnas.1218406109. Epub 2012 Nov 26.

Hadj-Bouziane F, Monfardini E, Guedj C, Gardechaux G, Hynaux C, Farnè A, Meunier M. (2014). The helmet head restraint system: a viable solution for resting state $\mathrm{fMRI}$ in awake monkeys. Neuroimage. Feb 1;86:536-43. doi: 10.1016/j.neuroimage.2013.09.068. Epub 2013 Oct 8.

Hagler DJ Jr, Sereno MI (2006). Spatial maps in frontal and prefrontal cortex. Neuroimage 29: 567-577.

Hagler Jr., D.J., Saygin, A.P., Sereno, M.I. (2006). Smoothing and cluster thresholding for cortical surfacebased group analysis of fMRI data. Neurolmage 33, 1093-1103

Hejja-Brichard Y, Rima S, Rapha E, Durand J-B, Cottereau BR. (2020). Stereomotion Processing in the Nonhuman Primate. Cerebral Cortex, 30: 4528-4543.

Helfrich RF, Becker HG, Haarmeier T (2013). Processing of Coherent Visual Motion in Topographically Organized Visual Areas in Human Cerebral Cortex. Brain Topogr 26(2): 247-63.

Kleinschmidt A, Thilo KV, Büchel C, Gresty MA, Bronstein AM, Frackowiak RS (2002). Neural correlates of visual-motion perception as object- or self-motion. Neuroimage 16(4): 873-882.

Koenderink JJ (1986). Optic flow. Vision Res 26: 161-168.

Kovács G, Raabe M, Greenlee MW (2008). Neural correlates of visually induced self-motion illusion in depth. Cereb Cortex 18: 1779-1787.

Lagae L, Maes H, Raiguel S, Xiao D-K, Orban GA. (1994). Responses of macaque STS neurons to optic flow components: a comparison of areas MT and MST. J Neurophysiol. 71:1597-1626.

Larsson J, Heeger DJ (2006). Two retinotopic visual areas in human lateral occipital cortex. J Neurosci 26(51): 13128-13142.

Morecraft RJ, Cipolloni PB, Stilwell-Morecraft KS, Gedney MT, Pandya DN (2004). Cytoarchitecture and cortical connections of the posterior cingulate and adjacent somatosensory fields in the rhesus monkey. J Comp Neurol 469(1): 37-69.

Morrone MC, Tosetti M, Montanaro D, Fiorentini A, Cioni G, Burr DC (2000). A cortical area that responds specifically to optic flow, revealed by fMRI. Nature Neurosci 3: 1322-1328.

Nakhla N, Korkian Y, Krause M R, Pack C C (2021). Neural Selectivity for Visual Motion in Macaque Area V3A. eNeuro, 8(1), 1-14. 
Nelissen K, Vanduffel W, Orban GA (2006). Charting the lower superior temporal region, a new motionsensitive region in monkey superior temporal sulcus. J Neurosci 26(22): 5929-47.

Oram, M. W., \& Perrett, D. I. (1994). Responses of anterior superior temporal polysensory (STPa) neurons to "biological motion" stimuli. Journal of Cognitive Neuroscience, 6(2), 99-116.

Orban G A, Fize D, Peuskens H, Denys K, Nelissen K, Sunaert S, Todd J, Vanduffel W (2003). Similarities and differences in motion processing between the human and macaque brain: evidence from fMRI. Neuropsychologia, 41, 1757-1768.

Orban GA, Lagae L, Verri A, Raiguel S, Xiao D, Maes H, Torre V (1992). First-order analysis of optical flow in monkey brain. Proc Natl Acad Sci USA 89(7): 2595-9.

Pitzalis S, Bozzacchi C, Bultrini A, Fattori P, Galletti C, Di Russo F (2013a). Parallel motion signals to the medial and lateral motion areas V6 and MT+. Neuroimage 67: 89-100.

Pitzalis S, Fattori P, Galletti C (2013b). The functional role of the medial motion area V6. Front Behav Neurosci 6: 91.

Pitzalis S, Fattori P, Galletti C (2015). The human cortical areas V6 and V6A. Vis Neurosci 32: E007.

Pitzalis S, Galletti C, Huang RS, Patria F, Committeri G, Galati G, Fattori P, Sereno MI (2006). Wide-field retinotopy defines human cortical visual area V6. J Neurosci 26: 7962-7973.

Pitzalis S, Sdoia S, Bultrini A, Committeri G, Di Russo F, Fattori P, Galletti C, Galati G (2013c). Selectivity to translational egomotion in human brain motion areas. PloS One, 8(4): e60241.

Pitzalis S, Sereno MI, Committeri G, Fattori P, Galati G, Patria F, Galletti C (2010). Human V6: the medial motion area. Cerebral Cortex 20(2): 411-24.

Pitzalis S, Sereno MI, Committeri G, Fattori P, Galati G, Tosoni A, Galletti C (2013d). The human homologue of macaque area V6A. Neuroimage 82: 517-530.

Pitzalis S, Serra C, Sulpizio V, Committeri G, de Pasquale F, Fattori P, Galletti C, Sepe R, Galati G. (2020). Neural bases of self- and object-motion in a naturalistic vision. Hum Brain Mapp doi: 10.1002/hbm.24862.

Pitzalis S, Serra C, Sulpizio V, Di Marco S, Fattori P, Galati G, Galletti C (2019). A putative human homologue of the macaque area PEc. Neuroimage Aug 10; 202:116092.

Pitzalis S, Strappini F, De Gasperis M, Bultrini A, Di Russo F (2012). Spatio-Temporal Brain Mapping of MotionOnset VEPs combined with fMRI and Retinotopic Maps. Plos One 7(4): e3577.

Previc FH, Liotti M, Blakemore C, Beer J, Fox P. (2000). Functional imaging of brain areas involved in the processing of coherent and incoherent wide field-of-view visual motion. Exp Brain Res. 131(4): 393-405.

Previc FH. (1998). The neuropsychology of 3-D space. Psychol Bull. 124(2):123--164.

Raffi M, Squatrito S, Maioli MG (2002). Neuronal responses to optic flow in the monkey parietal area Pec. Cereb Cortex 12: 639-646.

Raffi M, Maioli MG, Squatrito S. (2011). Optic flow direction coding in area PEc of the behaving monkey. Neuroscience. Oct 27;194:136-49.

Rosa MG, Tweedale R. (2001). The dorsomedial visual areas in New World and Old World monkeys: homology and function. Eur J Neurosci. 13(3):421--427.

Saito H, Yukie M, Tanaka K, Hikosaka K, Fukada Y, Iwai E (1986). Integration of direction signals of image motion in the superior temporal sulcus of the macaque monkey. J Neurosci 6(1): 145-57. 
Sereno MI, Dale AM, Reppas JB, Kwong KK, Belliveau JW, Brady TJ, Rosen BR, Tootell RBH (1995). Borders of multiple visual areas in humans revealed by functional magnetic resonance imaging. Science 268 : 889-893.

Sereno MI, Huang RS (2006). A human parietal face area contains aligned head-centered visual and tactile maps. Nat Neurosci 9(10): 1337-43.

Sereno MI, McDonald CT, Allman JM. (1994). Analysis of retinotopic maps in extrastriate cortex. Cereb Cortex. 4:601-620

Sereno MI, Pitzalis S, Martinez A (2001). Mapping of contralateral space in retinotopic coordinates by a parietal cortical area in humans. Science 294: 1350-1354.

Serra C, Galletti C, Di Marco S, Fattori P, Galati G, Sulpizio V, Pitzalis S. (2019). Egomotion-related visual areas respond to active leg movements. Hum Brain Mapp. Mar 28. doi: 10.1002/hbm.24589.

Siegel RM, Read HL. (1997). Analysis of optic flow in the monkey parietal area 7a. Cereb Cortex. 7(4):327-346.

Smith AT, Greenlee MW, Singh KD, Kraemer FM, Hennig J (1998). The processing of first- and second-order motion in human visual cortex assessed by functional magnetic resonance imaging (fMRI). J Neurosci 18: 3816-3830.

Smith AT, Wall MB, Thilo KV (2012). Vestibular inputs to human motion-sensitive visual cortex. Cereb Cortex 22(5): 1068-77.

Smith T, Wall MB, Williams AL, Singh KD (2006). Sensitivity to optic flow in human cortical areas MT and MST. Eur J Neurosci 23: 561-569.

Smith, A. T., Greenlee, M. W., DeAngelis, G. C., \& Angelaki, D. (2017). Distributed visual-vestibular processing in the cerebral cortex of man and macaque. Multisensory Research, 30(2), 91-120. https://doi.org/10. 1163/22134808-00002568

Strappini F, Gilboa E, Pitzalis S, Kay K, McAvoy M, Nehorai A, Snyder AZ (2017). Adaptive smoothing based on Gaussian processes regression increases the sensitivity and specificity of fMRI data. Hum Brain Mapp 38(3): 1438-1459.

Strappini F, Pitzalis S, Snyder AZ, McAvoy MP, Sereno MI, Corbetta M, Shulman GL (2015). Eye position modulates retinotopic responses in early visual areas: a bias for the straight-ahead direction. Brain Struct Funct 220(5): 2587-601.

Sulpizio V, Galati G, Fattori P, Galletti C, and Pitzalis S. (2020). A common neural substrate for processing scenes and egomotion-compatible visual motion. Brain structure and Function, in press.

Sunaert S, Van Hecke P, Marchal G, Orban G A (1999). Motion-responsive regions of the human brain. Exp Brain Res, 127:355-370.

Tanaka K, Fukada Y, Saito H (1989). Underlying mechanisms of the response specificity of expansion/contraction and rotation cells in the dorsal part of the MST area of the macaque monkey. J Neurophysiol 62: 642-656.

Tanaka K, Hikosaka K, Saito H, Yukie M, Fukada Y, Iwai E. (1986). Analysis of local and wide-field movements in the superior temporal visual areas of the macaque monkey. J Neurosci. Jan;6(1):134-44.

Tanaka K, Saito H (1989). Analysis of motion of the visual field by direction, expansion/contraction, and rotation cells clustered in the dorsal part of the medial superior temporal area of the macaque monkey. J Neurophysiol 62(3): 626-41.

Tanaka K1, Sugita Y, Moriya M, Saito H. (1993). Analysis of object motion in the ventral part of the medial superior temporal area of the macaque visual cortex. J Neurophysiol. Jan;69(1):128-42. 
Tootell RB, Hadjikhani N (2001). Where is 'dorsal V4' in human visual cortex? Retinotopic, topographic and functional evidence. Cereb Cortex 11(4): 298-311.

Tootell RB, Reppas JB, Kwong KK, Malach R, Born RT, Brady TJ, Rosen BR, Belliveau JW (1995). Functional analysis of human MT and related visual cortical areas using magnetic resonance imaging. J Neurosci 15: $3215-3230$.

Tootell RBH, Mendola JD, Hadjikhani NK, Ledden PJ, Liu AK, Reppas JB, Sereno MI, Dale AM (1997). Functional analysis of V3A and related areas in human visual cortex. J Neurosci 17: 7076-7078.

Van Essen DC, Maunsell HR, Bixby JL. (1981). The middle temporal visual area in the macaque: myeloarchitecture, connections, functional properties and topographic organization. J Comp Neurol. 199:293-326.

Van Oostende S, Sunaert S, Van Hecke P, Marchal G, Orban GA (1997). The kinetic occipital (KO) region in man: an fMRI study. Cereb Cortex 7(7): 690-701.

Vanduffel W, Fize D, Mandeville JB, Nelissen K, Van Hecke P, Rosen BR, Tootell RB, Orban GA. (2001). Visual motion processing investigated using contrast agent-enhanced $\mathrm{FMRI}$ in awake behaving monkeys. Neuron. Nov 20;32(4):565-77.

Vanduffel W, Zhu Q, Orban G.A. (2014). Monkey Cortex through fMRI Glasses. Neuron, 83(3): 533-50.

Wada A, Sakano Y, Ando H (2016). Differential Responses to a Visual Self-Motion Signal in Human Medial Cortical Regions Revealed by Wide-View Stimulation. Front Psychol 7: 309.

Wall MB, Lingnau A, Ashida H Smith AT (2008). Selective visual responses to expansion and rotation in the human MT complex revealed by functional magnetic resonance imaging adaptation. Eur J Neurosci 27: $2747-2757$.

Wall MB, Smith AT (2008). The Representation of Egomotion in the Human Brain. Curr Biol 18: 191-194.

Warren P A, \& Rushton, S. K. (2009a). Optic flow processing for the assessment of object movement during ego movement. Current Biology, 19(18), 1555-1560. https://doi.org/10.1016/j.cub.2009.07.057

Warren, P. A., \& Rushton S K (2009b). Perception of scene-relative object movement: Optic flow parsing and the contribution of monocular depth cues. Vision Research, 49(11), 1406-1419. https://doi.org/10.1016/j.visres.2009.01.016. 

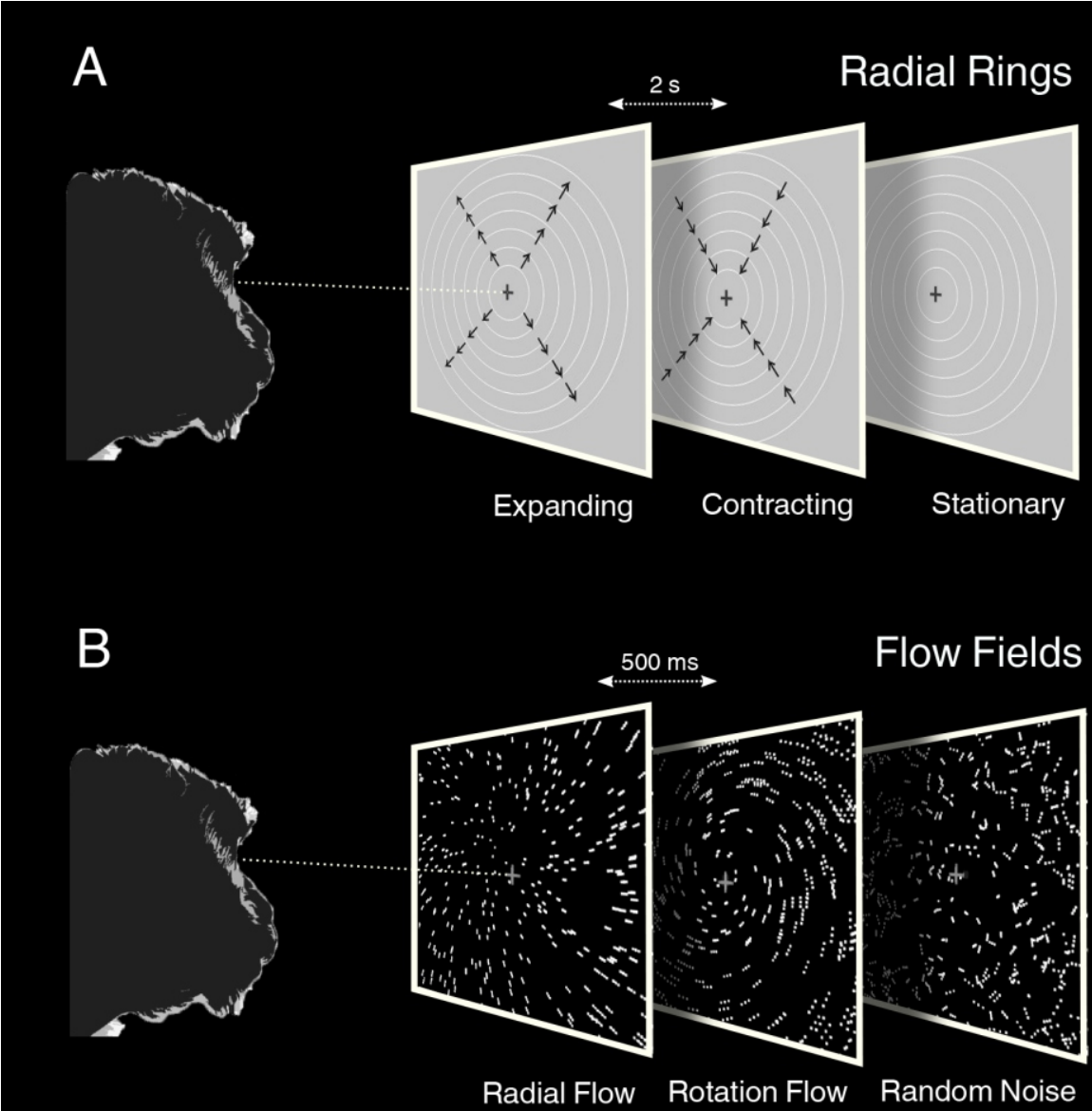

Radial Flow Rotation Flow Random Noise

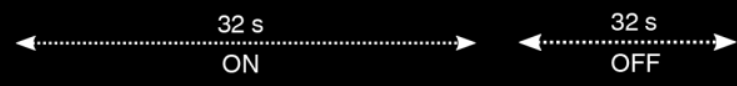




\section{Radial Rings}

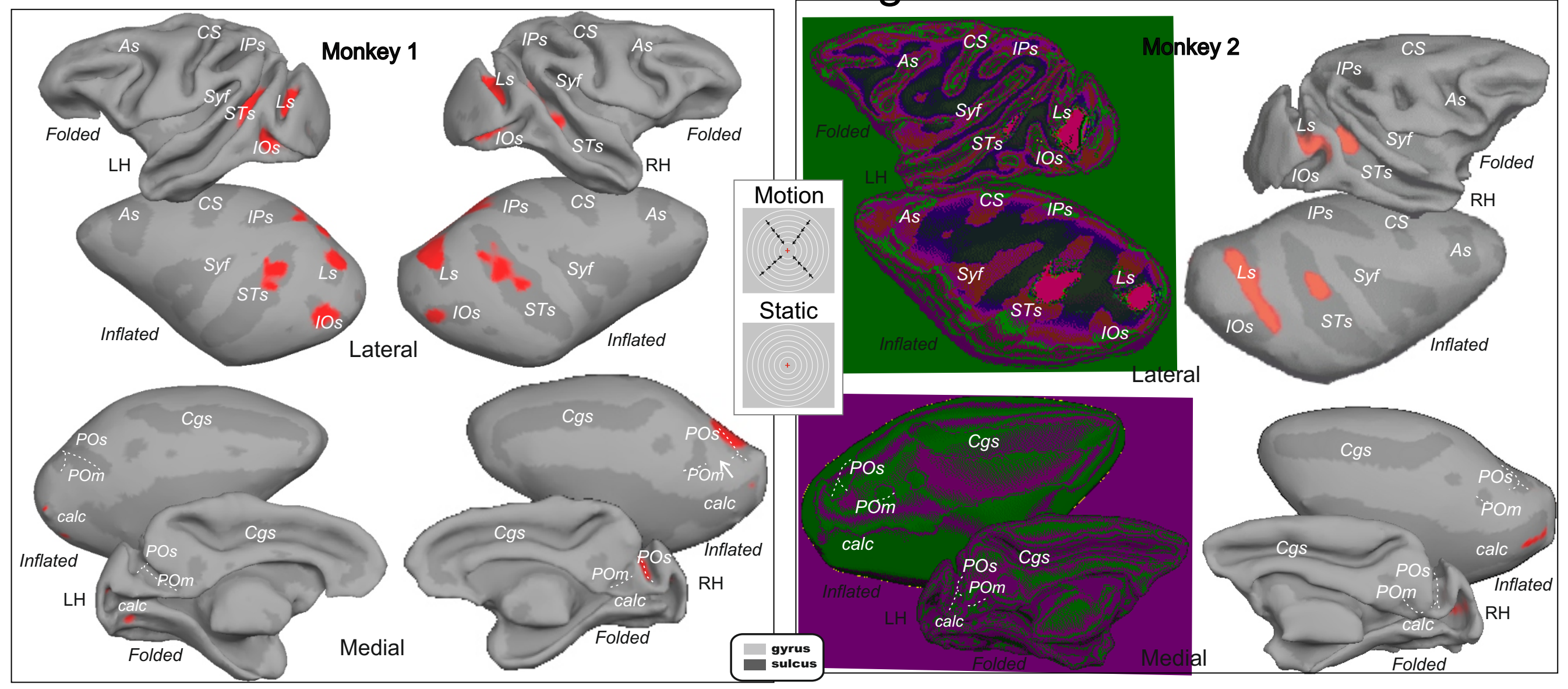




\section{Flow Fields}

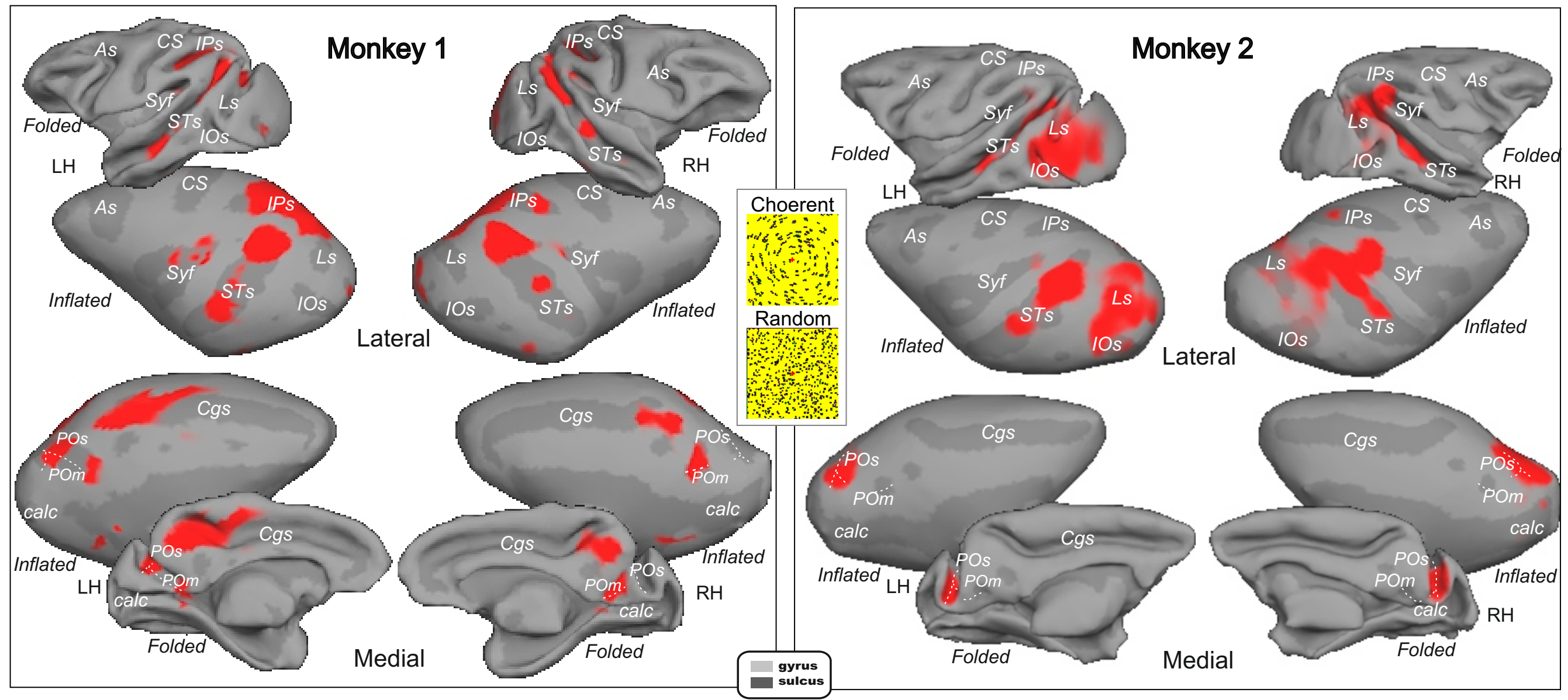




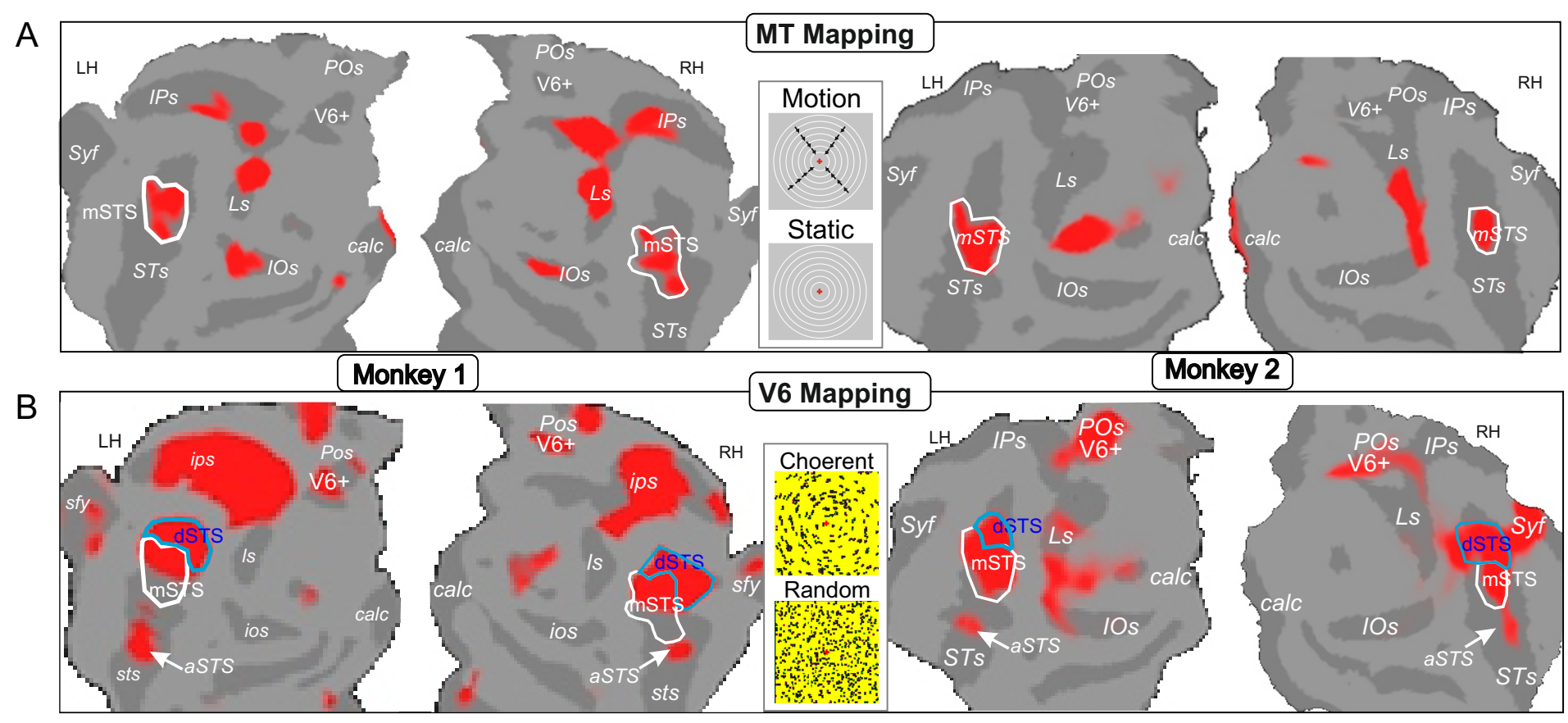


Monkey 1

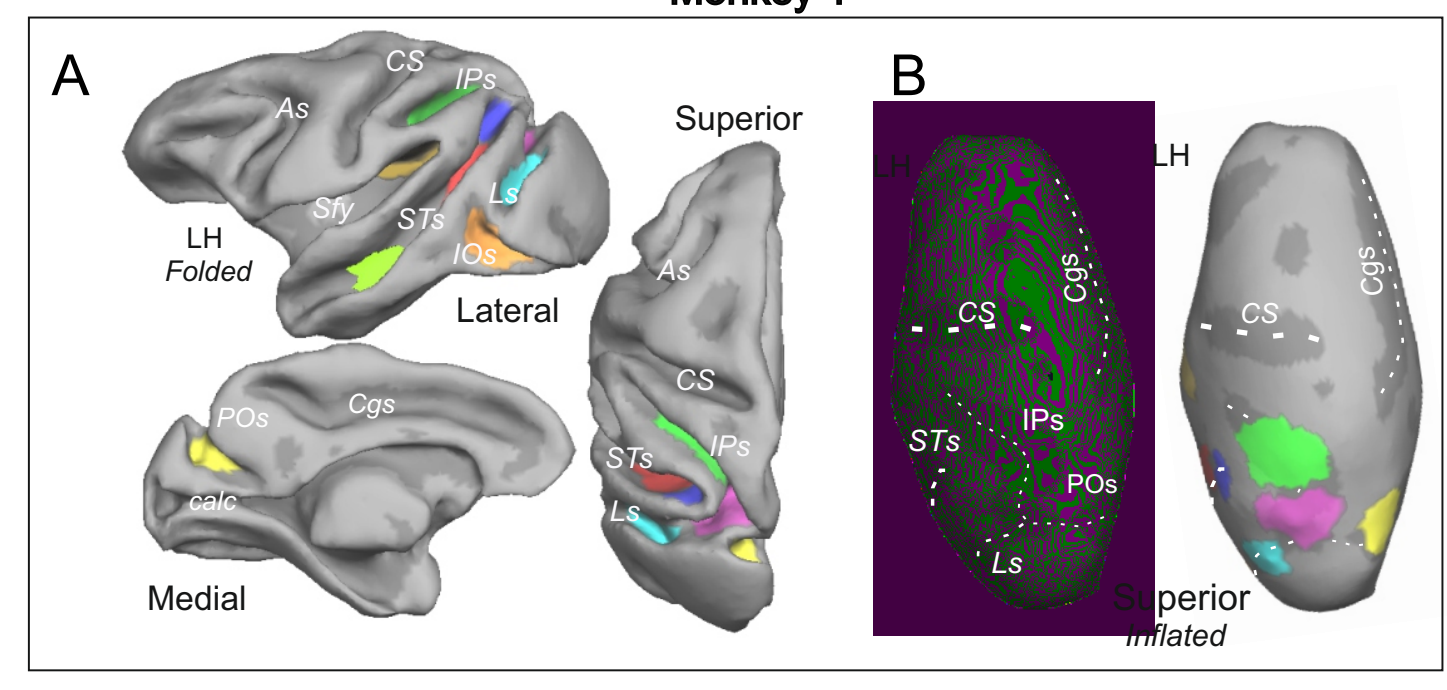

Monkey 2

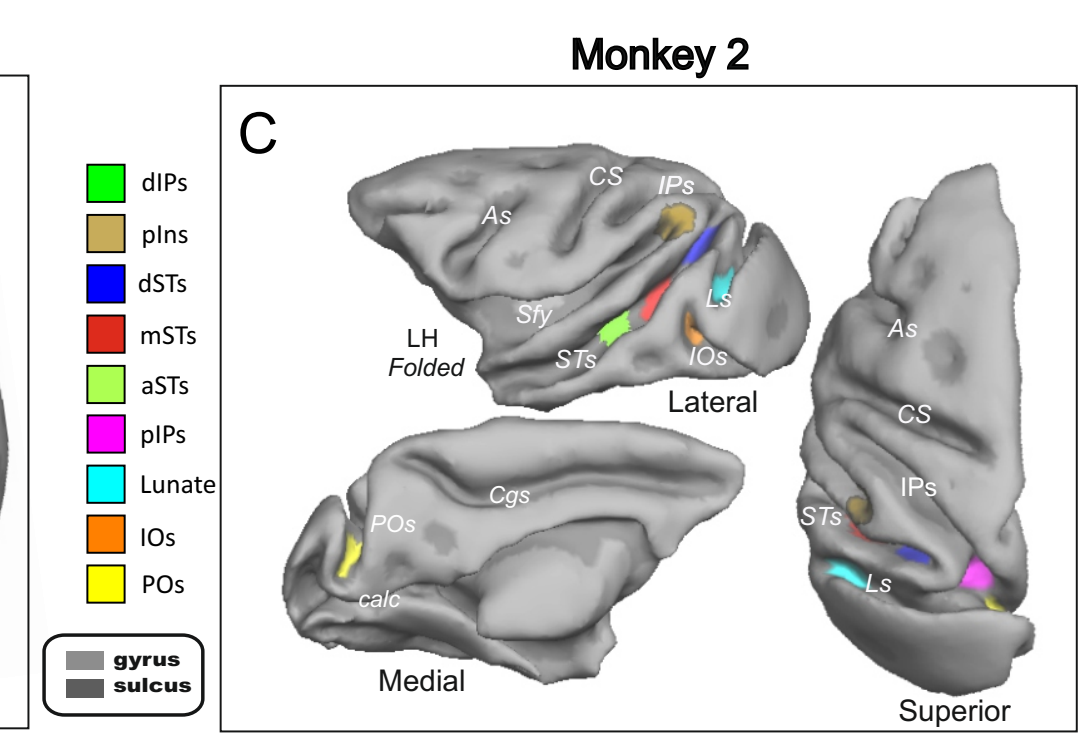



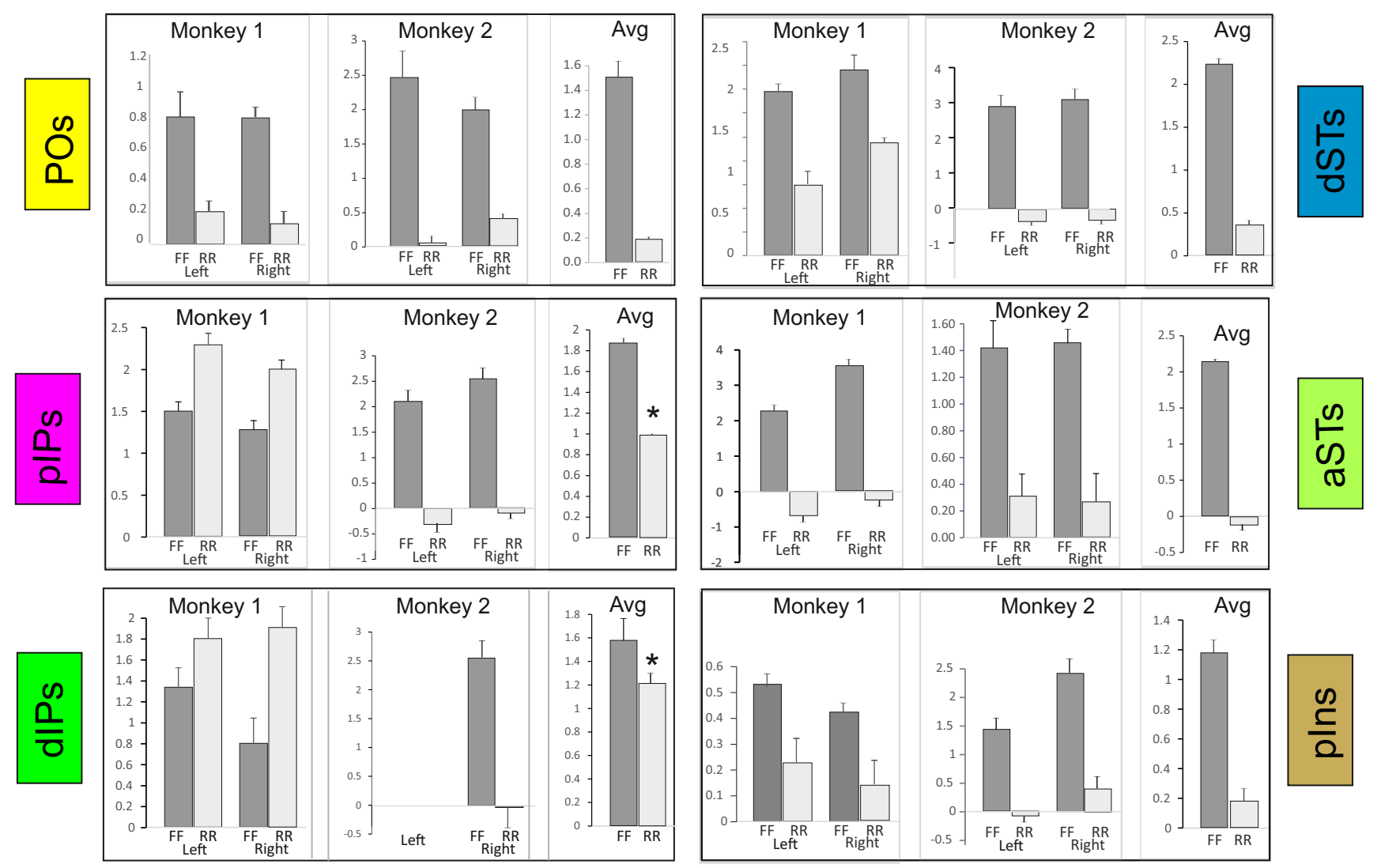

동 

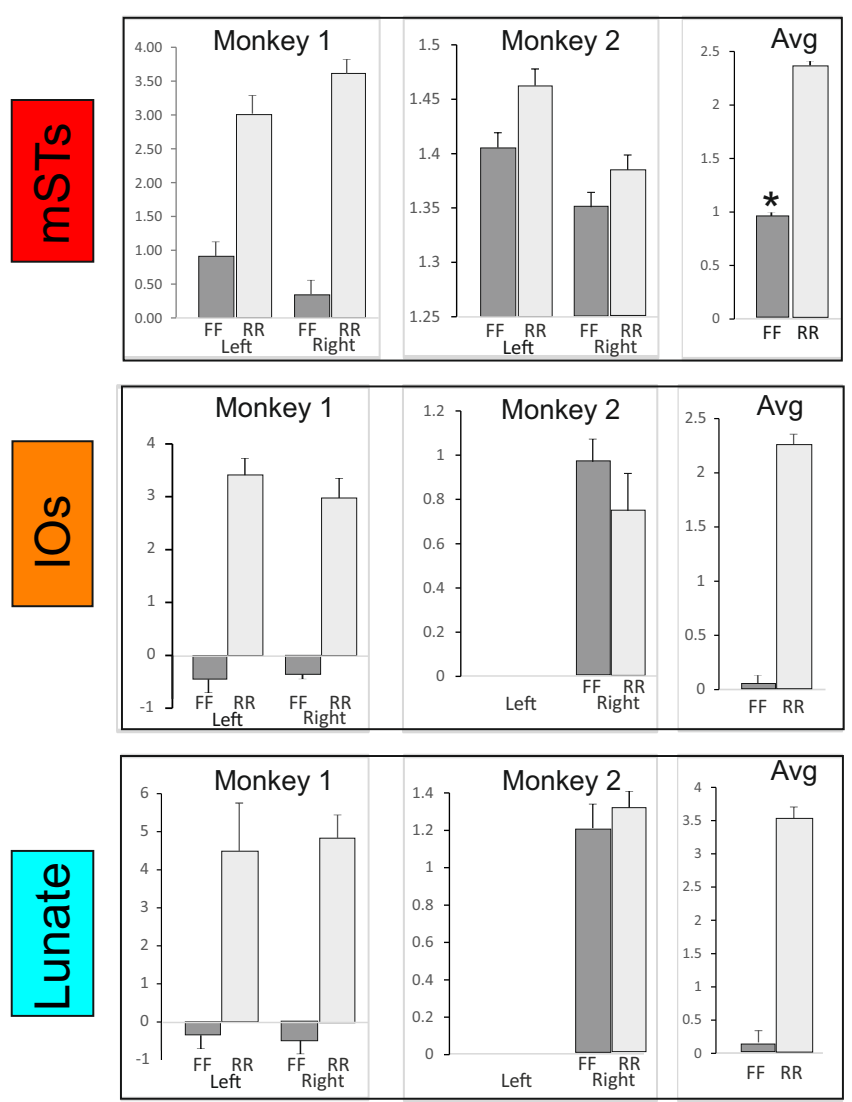


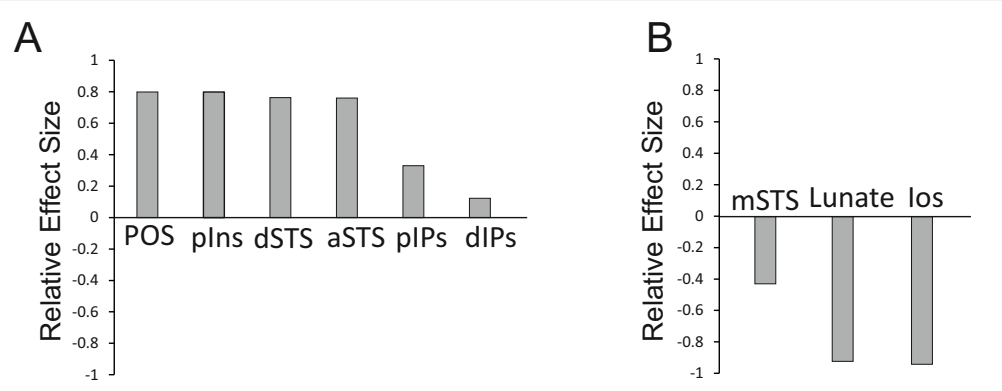

